\title{
Posicionamento Brasileiro sobre Hipertensão Arterial Resistente - 2020
}

Realização: Departamento de Hipertensão Arterial (DHA) da Sociedade Brasileira de Cardiologia

Conselho de Normatizações e Diretrizes (2020-2021): Brivaldo Markman Filho, Antonio Carlos Sobral Sousa, Aurora Felice Castro Issa, Bruno Ramos Nascimento, Harry Correa Filho, Marcelo Luiz Campos Vieira

Coordenador de Normatizações e Diretrizes (2020-2021): Brivaldo Markman Filho

Autores do Posicionamento: Juan Carlos Yugar-Toledo, ${ }^{10}$ Heitor Moreno Júnior, ${ }^{2}$ Miguel Gus, ${ }^{3}$ Guido Bernardo Aranha Rosito, ${ }^{4}$ Luiz César Nazário Scala, ${ }^{5}$ Elizabeth Silaid Muxfeldt, ${ }^{6}$ Alexandre Alessi, ${ }^{7}$ Andrea Araújo Brandão, ${ }^{8}$ Osni Moreira Filho, ${ }^{9}$ Audes Diógenes de Magalhães Feitosa, ${ }^{10}$ Oswaldo Passarelli Júnior, ${ }^{11}$ Dilma do Socorro Moraes de Souza, ${ }^{12}$ Celso Amodeo, ${ }^{11}$ Weimar Kunz Sebba Barroso, ${ }^{13}$ Marco Antônio Mota Gomes, ${ }^{14}$ Annelise Machado Gomes de Paiva, ${ }^{14}$ Eduardo Costa Duarte Barbosa, ${ }^{15}$ Roberto Dischinger Miranda, ${ }^{16}$ José Fernando Vilela-Martin, ${ }^{1}$ Wilson Nadruz Júnior, ${ }^{2}$ Cibele Isaac Saad Rodrigues, ${ }^{17}$ Luciano Ferreira Drager, ${ }^{18}$ Luiz Aparecido Bortolotto, ${ }^{18}$ Fernanda Marciano Consolim-Colombo, ${ }^{18}$ Márcio Gonçalves de Sousa, ${ }^{11}$ Flávio Antonio de Oliveira Borelli, ${ }^{11}$ Sérgio Emanuel Kaiser, ${ }^{8}$ Gil Fernando Salles, ${ }^{6}$ Maria de Fátima de Azevedo, ${ }^{19}$ Lucélia Batista Neves Cunha Magalhães, ${ }^{20}$ Rui Manoel dos Santos Póvoa, ${ }^{16}$ Marcus Vinícius Bolívar Malachias, ${ }^{21}$ Armando da Rocha Nogueira, ${ }^{22}$ Paulo César Brandão Veiga Jardim, ${ }^{23,24}$ Thiago de Souza Veiga Jardim²3

Faculdade Estadual de Medicina de São José do Rio Preto, ${ }^{1}$ São José do Rio Preto, SP - Brasil

Faculdade de Ciências Médicas da Universidade Estadual de Campinas, ${ }^{2}$ Campinas, SP - Brasil

Hospital Moinhos de Vento, ${ }^{3}$ Porto Alegre, RS - Brasil

Universidade Federal de Ciências da Saúde de Porto Alegre, ${ }^{4}$ Porto Alegre, RS - Brasil

Faculdade de Medicina da Universidade Federal de Mato Grosso, ${ }^{5}$ Cuiabá, MT - Brasil

Hospital Universitário Clementino Fraga Filho, Universidade Federal do Rio de Janeiro, ${ }^{6}$ Rio de Janeiro, RJ - Brasil

Universidade Federal do Paraná, ${ }^{7}$ Curitiba, PR - Brasil

Universidade do Estado do Rio de Janeiro, ${ }^{8}$ Rio de Janeiro, RJ - Brasil

Pontifícia Universidade Católica do Paraná, ${ }^{9}$ Curitiba, PR - Brasil

Universidade Federal de Pernambuco, ${ }^{10}$ Recife, PE - Brasil

Instituto Dante Pazzanese de Cardiologia, ${ }^{11}$ São Paulo, SP - Brasil

Faculdade de Medicina da Universidade Federal do Pará, ${ }^{12}$ Belém, PA - Brasil

Faculdade de Medicina da Universidade Federal de Goiás, ${ }^{13}$ Goiânia, GO - Brasil

Centro Universitário CESMAC, ${ }^{14}$ Maceió, AL - Brasil

Liga de Combate à Hipertensão de Porto Alegre, ${ }^{15}$ Porto Alegre, $R S$ - Brasil

Universidade Federal de São Paulo, ${ }^{16}$ São Paulo, SP - Brasil

Faculdade de Ciências Médicas e da Saúde Pontifícia Universidade Católica de são Paulo, ${ }^{17}$ São Paulo, SP - Brasil

Instituto do Coração do Hospital das Clínicas da Faculdade Medicina Universidade de São Paulo, ${ }^{18}$ São Paulo, SP - Brasil

Universidade Federal do Rio Grande do Norte, ${ }^{19}$ Natal, RN - Brasil

Faculdade de Tecnologia e Ciências da Universidade Federal da Bahia, ${ }^{20}$ Salvador, BA - Brasil

Faculdade de Ciências Médicas de Minas Gerais, ${ }^{21}$ Belo horizonte, MG - Brasil

Universidade Federal do Rio de Janeiro, ${ }^{22}$ Rio de Janeiro, $R J$ - Brasil

Faculdade de Medicina da Universidade Federal de Goiás, ${ }^{23}$ Goiânia, GO - Brasil

Hospital do Coração de Goiás, Goiânia, ${ }^{24} \mathrm{CO}$ - Brasil

Este posicionamento deverá ser citado como:

Yugar-Toledo JC, Moreno Júnior H, Gus M, Rosito GBA, Scala LCN, Muxfeldt ES, et al. Posicionamento Brasileiro sobre Hipertensão Arterial Resistente - 2020. Arq Bras Cardiol. 2020; 114(3):576-596

Nota: estes posicionamentos se prestam a informar e não a substituir o julgamento clínico do médico que, em última análise, deve determinar o tratamento apropriado para seus pacientes.

Correspondência: Sociedade Brasileira de Cardiologia - Av. Marechal Câmara, 360/330 - Centro - Rio de Janeiro - CEP: $20020-907$. E-mail: diretrizes@cardiol.br 


\section{Posicionamento}

\begin{tabular}{|c|c|c|c|c|c|c|c|}
\hline \multicolumn{8}{|c|}{$\begin{array}{l}\text { Declaração de potencial conflito de interesses dos autores/colaboradores do Posicionamento Brasileiro sobre Hipertensão Arterial } \\
\text { Se nos últimos } 3 \text { anos o autor/colaborador do Posicionamento: }\end{array}$} \\
\hline $\begin{array}{l}\text { Nomes Integrantes do } \\
\text { Posicionamento }\end{array}$ & $\begin{array}{l}\text { Participou de estudos } \\
\text { clínicos elou experimentais } \\
\text { subvencionados pela } \\
\text { indústria farmacêutica ou de } \\
\text { equipamentos relacionados } \\
\text { à diretriz em questão }\end{array}$ & $\begin{array}{l}\text { Foi palestrante } \\
\text { em eventos } \\
\text { ou atividades } \\
\text { patrocinadas } \\
\text { pela indústria } \\
\text { relacionados à } \\
\text { diretriz em questão }\end{array}$ & $\begin{array}{l}\text { Foi (é) membro } \\
\text { do conselho } \\
\text { consultivo } \\
\text { ou diretivo } \\
\text { da indústria } \\
\text { farmacêutica ou } \\
\text { de equipamentos }\end{array}$ & $\begin{array}{c}\text { Participou } \\
\text { de comitês } \\
\text { normativos } \\
\text { de estudos } \\
\text { científicos } \\
\text { patrocinados } \\
\text { pela indústria }\end{array}$ & $\begin{array}{l}\text { Recebeu auxílio } \\
\text { pessoal ou } \\
\text { institucional da } \\
\text { indústria }\end{array}$ & $\begin{array}{l}\text { Elaborou textos } \\
\text { científicos em } \\
\text { periódicos } \\
\text { patrocinados } \\
\text { pela indústria }\end{array}$ & $\begin{array}{c}\text { Tem } \\
\text { ações da } \\
\text { indústria }\end{array}$ \\
\hline Alexandre Alessi & Não & Não & Não & Não & Não & Não & Não \\
\hline Andrea Araújo Brandão & Não & $\begin{array}{l}\text { Abbott, EMS, } \\
\text { Merck }\end{array}$ & Não & Não & $\begin{array}{l}\text { Servier, } \\
\text { Medley }\end{array}$ & Servier, Abbott & Não \\
\hline $\begin{array}{l}\text { Annelise Machado Gomes } \\
\text { de Paiva }\end{array}$ & Não & Não & Não & Não & Não & Não & Não \\
\hline Armando da Rocha Nogueira & Não & Não & Não & Não & Não & Não & Não \\
\hline $\begin{array}{l}\text { Audes Diógenes de } \\
\text { Magalhães Feitosa }\end{array}$ & Não & Omron & Omron & Não & Não & Não & Não \\
\hline Celso Amodeo & Medtronic & Não & Não & Não & Novo Nordisk & Não & Não \\
\hline Cibele Isaac Saad Rodrigues & Não & Não & Não & Não & Não & Não & Não \\
\hline $\begin{array}{l}\text { Dilma do Socorro Moraes } \\
\text { de Souza }\end{array}$ & Não & Não & Não & Não & Não & Não & Não \\
\hline $\begin{array}{l}\text { Eduardo Costa Duarte } \\
\text { Barbosa }\end{array}$ & Não & Não & Não & Não & EMS, Servier & $\begin{array}{l}\text { EMS, Novartis, } \\
\text { Medley }\end{array}$ & Não \\
\hline Elizabeth Silaid Muxfeldt & Não & Não & Não & Não & Não & Não & Não \\
\hline $\begin{array}{l}\text { Fernanda Marciano } \\
\text { Consolim-Colombo }\end{array}$ & Não & Servier, Merck & Não & Não & $\begin{array}{l}\text { Servier, } \\
\text { Merck, } \\
\text { Daiichi } \\
\text { Sankyo }\end{array}$ & Servier, Merck & Não \\
\hline $\begin{array}{l}\text { Flavio Antonio de Oliveira } \\
\text { Borelli }\end{array}$ & Não & Não & Não & Não & Não & Libbs & Não \\
\hline Gil Fernando Salles & Não & Não & Não & Não & Não & Não & Não \\
\hline $\begin{array}{l}\text { Guido Bernardo Aranha } \\
\text { Rosito }\end{array}$ & Não & Não & Não & Não & Não & Não & Não \\
\hline Heitor Moreno Júnior & Não & Não & Não & Não & Não & Não & Não \\
\hline José Fernando Vilela-Martin & Não & Não & Não & Não & Não & Não & Não \\
\hline Juan Carlos Yugar-Toledo & Não & Não & Não & Não & Não & Não & Não \\
\hline Luciano Ferreira Drager & Não & Não & Não & Não & Não & Não & Não \\
\hline $\begin{array}{l}\text { Lucélia Batista Neves Cunha } \\
\text { Magalhães }\end{array}$ & Não & Não & Não & Não & Não & Não & Não \\
\hline Luiz Aparecido Bortolotto & Não & Não & Não & Não & Não & Servier, Merck & Não \\
\hline Luiz César Nazário Scala & Não & Não & Não & Não & Não & Não & Não \\
\hline Márcio Gonçalves de Sousa & Não & Não & Não & Não & Não & Não & Não \\
\hline Marco Antônio Mota Gomes & Não & Não & $\begin{array}{c}\text { Servier, Torrent, } \\
\text { Abbott, Omron, } \\
\text { Novartis, } \\
\text { Astrazeneca }\end{array}$ & Não & $\begin{array}{l}\text { Servier, } \\
\text { Torrent, } \\
\text { Abbott, } \\
\text { Omron, } \\
\text { Novartis, } \\
\text { Astrazeneca }\end{array}$ & $\begin{array}{c}\text { Servier, } \\
\text { Torrent, } \\
\text { Abbott, Omron, } \\
\text { Novartis, } \\
\text { Astrazeneca }\end{array}$ & Não \\
\hline $\begin{array}{l}\text { Marcus Vinícius Bolívar } \\
\text { Malachias }\end{array}$ & Não & $\begin{array}{l}\text { Abbott, Biolab, } \\
\text { Libbs, Novo } \\
\text { Nordisk, Takeda }\end{array}$ & Não & Não & Não & $\begin{array}{c}\text { Abbott, Biolab, } \\
\text { Farmoquímica, } \\
\text { Libbs, Novo } \\
\text { Nordisk }\end{array}$ & Não \\
\hline Maria de Fátima de Azevedo & Não & Não & Não & Não & Não & Não & Não \\
\hline Miguel Gus & Não & Não & Não & Não & Não & Não & Não \\
\hline Osni Moreira Filho & Não & Servier, Merck & Não & Não & Não & Não & Não \\
\hline
\end{tabular}




\section{Posicionamento}

\begin{tabular}{|c|c|c|c|c|c|c|c|}
\hline Oswaldo Passarelli Júnior & Não & Não & Não & Não & Não & Não & Não \\
\hline $\begin{array}{l}\text { Paulo César Brandão Veiga } \\
\text { Jardim }\end{array}$ & Não & Não & Não & Não & Não & $\begin{array}{l}\text { Biolab, Aché, } \\
\text { Libbs }\end{array}$ & Não \\
\hline Roberto Dischinger Miranda & Não & Não & Não & Não & Não & Não & Não \\
\hline $\begin{array}{l}\text { Rui Manoel dos Santos } \\
\text { Póvoa }\end{array}$ & Não & Não & Não & Não & Não & Não & Não \\
\hline Sérgio Emanuel Kaiser & Não & Não & Não & Não & Não & Novartis & Não \\
\hline $\begin{array}{l}\text { Thiago de Souza Veiga } \\
\text { Jardim }\end{array}$ & Não & Torrent & Não & Não & $\begin{array}{l}\text { Libbs, Torrent, } \\
\text { Novartis }\end{array}$ & Torrent & Não \\
\hline Weimar Kunz Sebba Barroso & $\begin{array}{l}\text { Amgen, AstraZeneca, } \\
\text { Torrent, EMS, Novartis }\end{array}$ & Não & Não & Não & $\begin{array}{c}\text { EMS, Sandoz, } \\
\text { Servier, } \\
\text { Novartis }\end{array}$ & $\begin{array}{c}\text { Medley, } \\
\text { Sandoz, EMS }\end{array}$ & Não \\
\hline Wilson Nadruz Júnior & Não & Não & Não & Não & Não & Não & Não \\
\hline
\end{tabular}




\section{Posicionamento}

\section{Sumário}

1. Definição e Epidemiologia 580

1.1. Definição/Novos Conceitos

.580

1.2. Controle da Hipertensão Arterial no Brasil e no Mundo ....................558

1.3. Incidência e Prevalência de Hipertensão Arterial Resistente .............580

1.4. Fatores Relacionados à Hipertensão Arterial Resistente ..................580

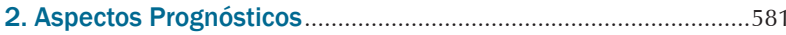

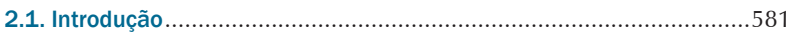

2.2. Pressão Arterial de Consultório e Monitoramento Ambulatorial da

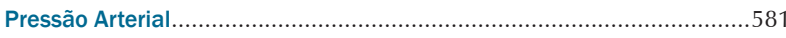

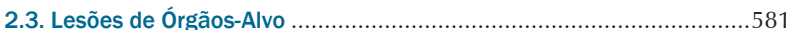

2.3.1. Pressão Arterial Central e Rigidez Arterial ...................................581

2.3.2. Hipertrofia Ventricular Esquerda ……....................................581

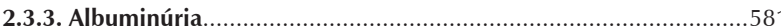

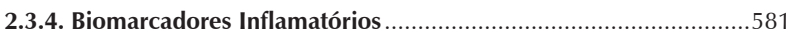

3. Fluxograma de Avaliação de Hipertensão Arterial

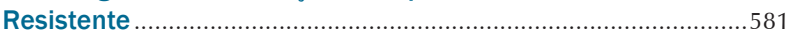

3.1. Fluxograma na Abordagem Diagnóstica da Hipertensão Arterial

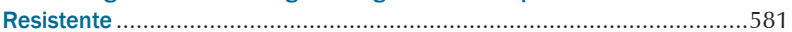

4. Medida da Pressão Arterial..........................................................582

4.1. Pressão Arterial de Consultório na Hipertensão Arterial

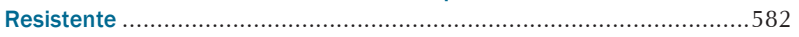

4.2. Monitoramento Ambulatorial da Pressão Arterial em Hipertensão

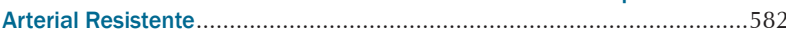

4.3. Monitoramento Residencial da Pressão Arterial e Automedida da

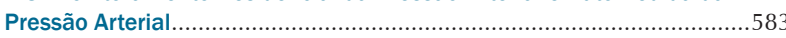

4.4. Medida da Pressão Arterial Central ...............................................583

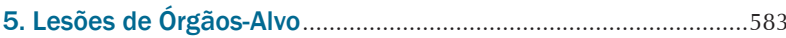

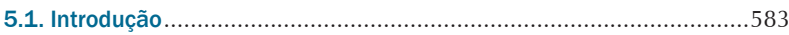

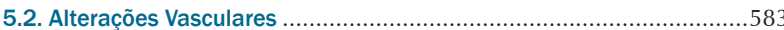

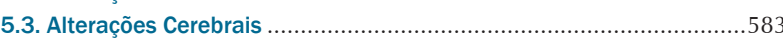

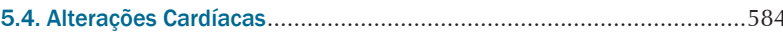

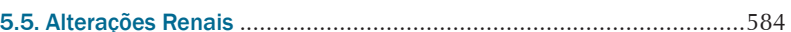

6. Fenótipo do Paciente com Hipertensão Arterial Resistente....585

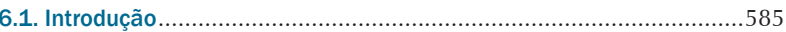

6.2. Fenótipo do Paciente com Hipertensão Arterial Resistente ................585

6.3. Fenótipo da Hipertensão Arterial Resistente Controlada e da Não

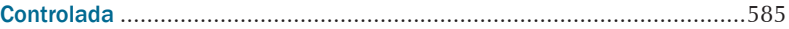

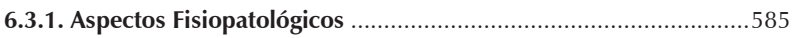

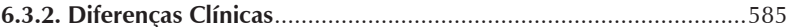

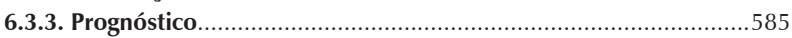

6.4. Fenótipo do Paciente com Hipertensão Arterial Refratária .................585

7. Causas Secundárias de Hipertensão Arterial Resistente ..........586

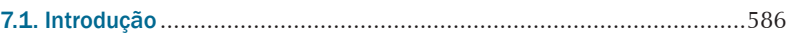

7.2. Hipertensão Arterial Secundária de Causas Não Endócrinas..............586

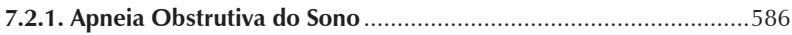

7.2.2. Doença do Parênquima Renal ....................................................586

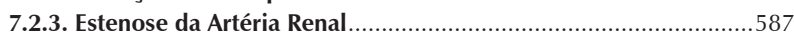

7.3. Hipertensão Arterial Secundária de Causas Endócrinas.....................587

7.3.1. Hiperaldosteronismo Primário........................................................ 587

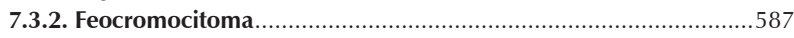

7.3.3. Hipotireoidismo e Hipertireoidismo ………………...................58

8. Tratamento Não Farmacológico ......................................................58

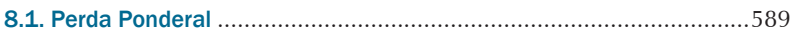

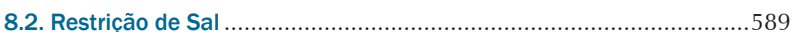

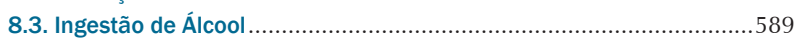

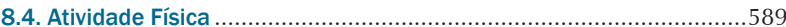

9. Tratamento Farmacológico da Hipertensão Arterial

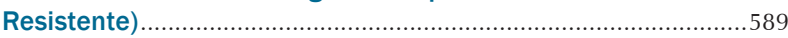

10. Novos Tratamentos da Hipertensão Arterial Resistente .....590

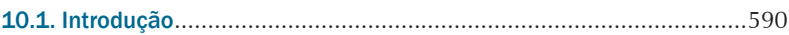

10.2. Estimulação Direta do Seio Carotídeo ...........................................590

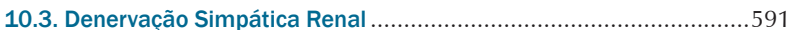

10.4. Uso de Pressão Positiva Contínua em Vias Aéreas ..........................591

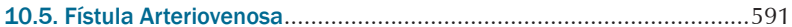

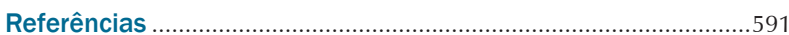




\section{Definição e Epidemiologia}

Coordenador: Heitor Moreno Júnior.

Autores: Juan Carlos Yugar-Toledo, Heitor Moreno Júnior, Miguel Gus, Guido Bernardo Aranha Rosito e Luiz César Nazário Scala.

\subsection{Definição/Novos Conceitos}

A hipertensão arterial resistente (HAR) é definida quando a pressão arterial (PA) permanece acima das metas recomendadas com o uso de três anti-hipertensivos de diferentes classes, incluindo um bloqueador do sistema reninaangiotensina (inibidor da enzima conversora da angiotensina [IECA] ou bloqueador do receptor de angiotensina [BRA]), um bloqueador dos canais de cálcio (BCC) de ação prolongada e um diurético tiazídico (DT) de longa ação em doses máximas preconizadas e toleradas, administradas com frequência, dosagem apropriada e comprovada adesão.

Outros fármacos podem ser associados aos primeiros em caso de falha deles (antagonistas da aldosterona, betabloqueadores e $\alpha$-metildopa); entretanto, especialistas conflitam sobre o assunto em alguns pontos referentes à dose/potência, embora a maior discussão seja quanto ao uso da clortalidona ou da hidroclorotiazida como principais DT. ${ }^{1}$

Nesta definição está incluído o subgrupo de pacientes hipertensos resistentes, cuja PA é controlada com quatro ou mais medicamentos anti-hipertensivos, chamada de HAR controlada (HAR-C). ${ }^{2,3}$ A classificação da doença em HAR-C e HAR não controlada (HAR-NC) ${ }_{1}^{4}$ incluindo a HAR refratária (HAR-Ref), um fenótipo extremo de HAR-NC em uso de cinco ou mais anti-hipertensivos, ${ }^{5}$ é uma proposta que ganha espaço na literatura. ${ }^{6,7}$

Assim, HAR-NC é definida como uma PA que permanece acima do nível desejado (140/90 mmHg), apesar do uso concomitante de quatro ou mais agentes anti-hipertensivos de diferentes classes e um quarto fármaco, que geralmente é um antagonista do receptor mineralocorticoide ou um bloqueador simpático central (Quadro 1).

Quadro 1 - Classificação da hipertensão arterial resistente

\begin{tabular}{lcc}
\hline & $\begin{array}{c}\text { Número de anti- } \\
\text { hipertensivos }\end{array}$ & \\
\hline N & & \\
$\begin{array}{l}\text { Hlpertensão resistente } \\
\text { controlada }\end{array}$ & 6 & $\begin{array}{c}\text { Hipertensão resistente } \\
\text { não controlada }\end{array}$ \\
& 5 & \\
\cline { 2 - 3 } & 4 & Hipertensão resistente \\
\hline$<140 / 90$ & 2 & $\geq 140 / 90$ \\
\hline Normotensão & 1 & Hipertensão \\
\hline
\end{tabular}

1.2. Controle da Hipertensão Arterial no Brasil e no Mundo

A análise de 135 estudos populacionais com 1 milhão de indivíduos indica que $31,1 \%$ da população adulta é hipertensa (IC 95\%; 30 a 32\%), com valor estimado em 28,5 e 31,5\% nos países de maior e menor condição socioeconômica, respectivamente. O controle pressórico varia dependendo das condições socioeconômicas, chegando a $28,4 \%$ nos países mais desenvolvidos e apenas $7,7 \%$ naqueles com menor grau de desenvolvimento. ${ }^{8}$ No Brasil, a taxa de controle varia de 10,4 a $35,2 \%$ nas populações estudadas em três regiões do país. ${ }^{9}$

Um estudo envolvendo 291 centros das cinco regiões brasileiras e 2.810 pacientes avaliou a taxa de controle conforme perfil de risco e metas pressóricas. Para pacientes de menor risco e meta $<140 / 90 \mathrm{mmHg}$, o controle foi de $61,7 \%$, enquanto para hipertensos de alto risco com meta $<130 / 80 \mathrm{mmHg}$ o valor correspondente foi de $41,8 \% .^{10}$

\subsection{Incidência e Prevalência de Hipertensão Arterial Resistente}

A prevalência de HAR no mundo é estimada entre 10 e 20\% dos hipertensos, o que significa aproximadamente 200 milhões de hipertensos resistentes. ${ }^{11}$ A variabilidade deve-se, principalmente, à diferença de critérios para HAR e às características das populações estudadas.

O National Health and Nutrition Examination Survey (NHANES) demonstrou uma prevalência de cerca de $9 \%$ dos hipertensos tendo HAR, correspondendo a 12,8\% daqueles que utilizam anti-hipertensivos nos EUA. ${ }^{12}$

No entanto, a real prevalência de HAR não é conhecida. Uma metanálise de Achelrod et al. ${ }^{11}$ avaliando populações de hipertensos tratados encontrou uma prevalência de 13,72\% (IC 95\%; 11,19 a 16,24\%), de acordo com 20 estudos observacionais, e 16,32\% (IC 95\%; 10,68 a 21,95\%) para quatro ensaios clínicos randomizados. ${ }^{11}$ No Brasil, um estudo multicêntrico e utilizando o monitoramento ambulatorial da pressão arterial (MAPA) (ReHOT study) mostrou uma prevalência de HAR de $11,7 \% .^{13}$

Daugherty et al. ${ }^{14}$ analisaram a incidência de HAR em 205.750 hipertensos que iniciaram tratamento antihipertensivo entre 2002 e 2006. A taxa foi de 1,9\% com um ano e meio de acompanhamento (0,7 por 100 pacientes ao ano), acarretando um risco cardiovascular (CV) 1,47 maior em 3,8 anos. ${ }^{14}$

\subsection{Fatores Relacionados à Hipertensão Arterial Resistente}

A HAR é mais prevalente em idosos, obesos e afrodescendentes, bem como em pacientes com hipertrofia ventricular esquerda, diabetes melito, nefropatia crônica, síndrome metabólica, elevada ingestão de álcool e/ou sal e sedentarismo. ${ }^{1,15-17}$ Os fatores relacionados à HAR abrangem inúmeros aspectos, como: 1) diagnósticos: técnica inadequada de aferição da PA, efeito do avental branco; $;^{1,15}$ 2) causais: maior sensibilidade ao sal, expansão volêmica por ingestão 
excessiva de sal ou doença renal crônica (DRC), uso de anti-inflamatórios não hormonais, esteroides anabólicos, contraceptivos orais, simpaticomiméticos (descongestionantes nasais, inibidores do apetite, cocaína), quimioterápicos, antidepressivos, eritropoietina, imunodepressores, álcool; ${ }^{1,15}$ 3) causas secundárias de hipertensão, destacando-se: hiperaldosteronismo primário, apneia obstrutiva do sono (AOS), DRC, estenose de artéria renal, doenças tireóideas; ${ }^{15}$ 4) terapêuticos: fármacos inapropriados ou em doses insuficientes, inércia médica, baixa aderência. ${ }^{16,17}$ Tanto a hipertensão sistólica quanto a diastólica podem ser resistentes, sendo a primeira mais prevalente. ${ }^{1}$

\section{Aspectos Prognósticos}

Coordenadora: Elizabeth Silaid Muxfeldt.

Autores: Alexandre Alessi, Andrea Araújo Brandão, Osni Moreira Filho e Elizabeth Silaid Muxfeldt.

\subsection{Introdução}

A HAR está relacionada a uma alta morbimortalidade $\mathrm{CV}$, apresentando um risco $47 \%$ maior de desenvolver eventos CV quando comparados aos hipertensos em geral. ${ }^{14}$

\subsection{Pressão Arterial de Consultório e Monitoramento Ambulatorial da Pressão Arterial}

A HAR verdadeira, diagnosticada pelo MAPA, tem o dobro do risco $\mathrm{CV}$ em comparação à HAR relacionada a efeito do avental branco. ${ }^{18}$ De modo geral, as médias de PA obtidas nos três períodos do MAPA são fortes preditoras de risco $\mathrm{CV}$, enquanto a PA de consultório não demonstrou nenhum valor prognóstico. ${ }^{18,19}$ Estudos longitudinais destacaram a PA no sono elevada e a ausência de descenso noturno como importantes preditores de risco CV. ${ }^{18-20}$ A importância prognóstica do comportamento noturno da PA também já foi demonstrada em metanálises. ${ }^{21}$

\subsection{Lesões de Órgãos-Alvo}

\subsubsection{Pressão Arterial Central e Rigidez Arterial}

A velocidade de onda de pulso (VOP) tem valor preditivo independente em vários subgrupos de pacientes hipertensos. ${ }^{22}$ Nos resistentes, foi observado maior rigidez arterial que nos controlados, sendo um marcador de prognóstico e de resposta terapêutica anti-hipertensiva. ${ }^{23} \mathrm{Em}$ hipertensos existe um valor aditivo quando a VOP é agregada a escores de risco CV. ${ }^{24}$

\subsubsection{Hipertrofia Ventricular Esquerda}

O diagnóstico eletrocardiográfico de hipertrofia ventricular esquerda (HVE) foi preditor de risco para doença coronariana (índice de Cornell) e cerebrovascular (índice de SokolowLyon), e a regressão desses dois índices reduziu o risco de eventos CV em 35 e 40\%, respectivamente. ${ }^{25}$

\subsubsection{Albuminúria}

Na HAR há implicações prognósticas tanto da albuminúria inicial como do seu padrão evolutivo. Em uma grande coorte prospectiva com 531 hipertensos resistentes, a albuminúria moderadamente elevada (AME) inicial foi um preditor independente de eventos compostos e mortalidade total. ${ }^{26}$ Uma nova análise do mesmo grupo, porém envolvendo 1.048 pacientes, identificou que a AME aumenta em $40 \%$ o risco de eventos CV fatais e não fatais e de mortalidade total. ${ }^{27}$

Evolutivamente, a persistência da AME em 2 anos foi um fator de risco para eventos $\mathrm{CV}$, enquanto a normoalbuminúria persistente foi fator de proteção. ${ }^{26}$ Outra coorte envolvendo 143 pacientes com HAR, analisados no momento basal e após 6 anos de seguimento, mostrou que o desenvolvimento de AME ou a sua persistência estavam relacionados a maior risco de eventos CV. Em contrapartida, a persistência de normoalbuminúria, ou a regressão da $\mathrm{AME}$, se associou a menor risco de eventos maiores. ${ }^{28}$

\subsubsection{Biomarcadores Inflamatórios}

A proteína C reativa elevada foi preditora independente de doença coronariana e cerebrovascular, sendo um marcador mais importante para hipertensos resistentes mais jovens, obesos, com MAPA não controlado e padrão não dipper (descenso noturno ausente ou atenuado). ${ }^{29}$

\section{Fluxograma de Avaliação de Hipertensão Arterial Resistente}

Coordenador: Audes Diógenes de Magalhães Feitosa.

Autores: Oswaldo Passarelli Júnior, Dilma do Socorro Moraes de Souza e Audes Diógenes de Magalhães Feitosa.

\subsection{Fluxograma na Abordagem Diagnóstica da Hipertensão Arterial Resistente}

Diante da suspeita clínica de HAR, é necessário verificar a confirmação diagnóstica, e a primeira etapa na investigação é a exclusão das causas de pseudorresistência, tais como falta de adesão ao tratamento (farmacológico e não farmacológico), posologia inadequada, técnica imprópria de aferição da PA e efeito do avental branco ${ }^{1}$ (Figura 1). O MAPA e o monitoramento residencial da pressão arterial (MRPA) são os exames para confirmação do controle inadequade da PA. ${ }^{30-32}$

Uma vez afastada a pseudorresistência, confirma-se a existência da HAR e inicia-se uma investigação diagnóstica com exames específicos, conforme a orientação das Diretrizes de Hipertensão em relação ao comprometimento de lesões em órgãos-alvo e hipertensão secundária. ${ }^{33,34} \mathrm{~A}$ ocorrência de comorbidades associadas deve ser detectada com exames especializados de acordo com a suspeita clínica.

A medida da PA fora do consultório é fundamental, pois ela costuma ser mais elevada em relação à medida domiciliar; desse modo, o efeito do avental branco é frequente nessa população. A adesão ao tratamento é sempre um grande desafio, especialmente nos serviços públicos.

Alguns problemas relacionados aos pacientes podem ocorrer, como: rejeição a número excessivo de fármacos em posologias complexas (muitas tomadas e comprimidos), efeitos colaterais dos medicamentos, problemas socioculturais e desconhecimento da história natural da doença, além de 


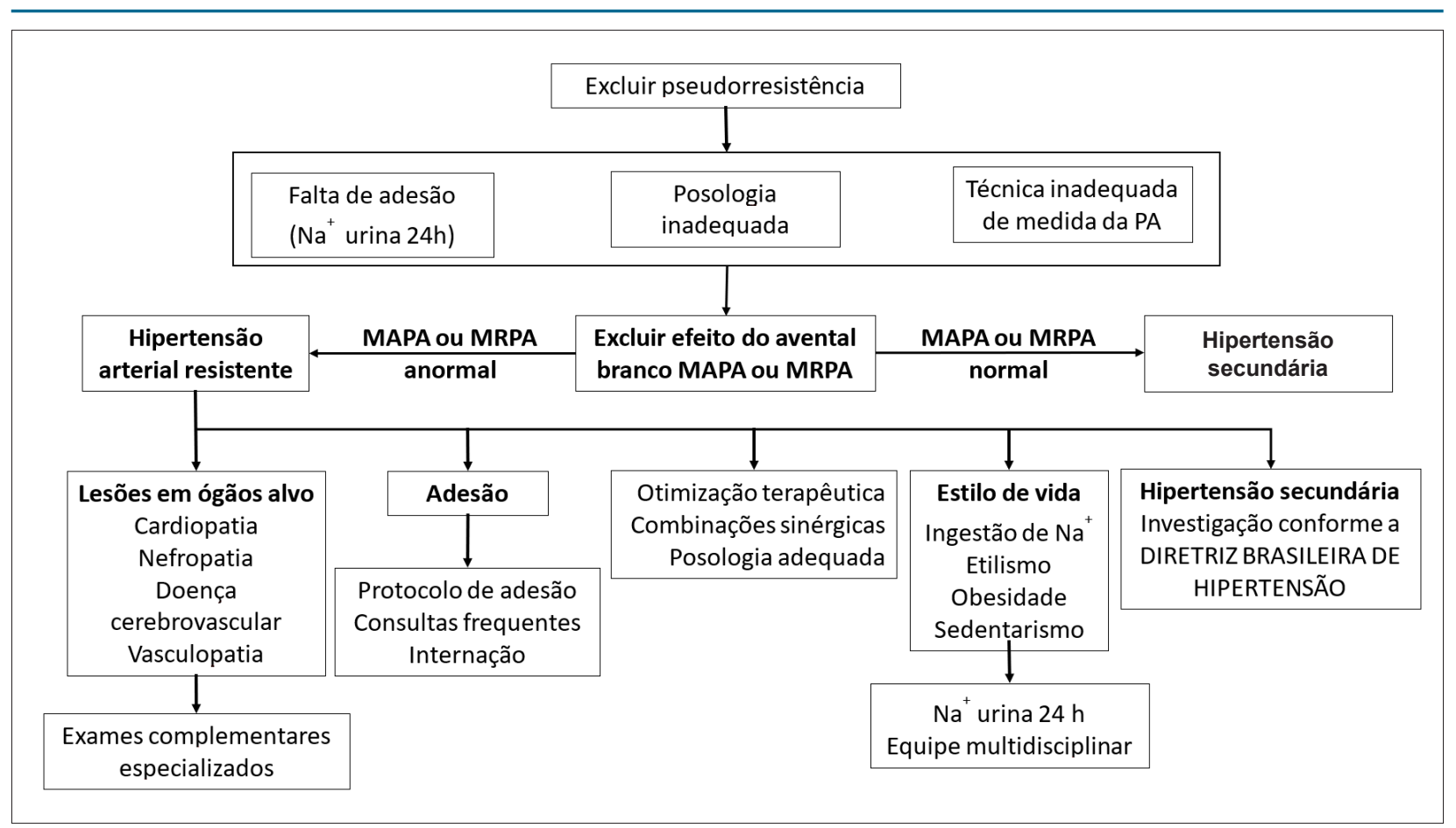

Figura 1 - Fluxograma de avaliação da hipertensão arterial resistente. MAPA: monitoramento ambulatorial da pressão arterial; MRPA: monitoramento residencial da pressão arterial; $\mathrm{Na+}$ : sódio; PA: pressão arterial.

outros referentes ao médico, como: relação médico-paciente ruim, posologias não sinérgicas ou doses equivocadas e omissão ou desconhecimento na investigação das causas secundárias tratáveis. Um problema relacionado aos serviços de saúde pode ser a dificuldade de acesso aos médicos, medicamentos e exames complementares.

Todos esses fatores dificultam a adesão ao tratamento farmacológico e não farmacológico; por isso, devem ser verificados e contornados.

A ingesta de sal precisa sempre ser conferida, se possível com a verificação do sódio em urina de 24 horas, pois frequentemente a ingestão é excessiva em função do consumo de alimentos industrializados e do desconhecimento dos pacientes em relação ao consumo excessivo de sal.

A otimização terapêutica deve ser realizada preferencialmente com o mesmo médico, por um período mínimo de 6 meses, para o fortalecimento da relação médicopaciente. Somam-se a isso orientação constante em relação ao estilo de vida saudável e a verificação contínua da adesão ao tratamento, com esquemas posológicos sinérgicos e ajustes medicamentosos adequados, respeitando a existência de comorbidades que indiquem ou contraindiquem determinada classe de fármaco anti-hipertensivo.

\section{Medida da Pressão Arterial \\ Coordenador: Celso Amodeo.}

Autores: Weimar Kunz Sebba Barroso, Marco Antônio Mota Gomes, Annelise Machado Gomes de Paiva e Eduardo Costa Duarte Barbosa.

\subsection{Pressão Arterial de Consultório na Hipertensão Arterial Resistente}

A verificação da PA de consultório, apesar de não ser diagnóstica para HAR, deve ser realizada, e o procedimento de aferição precisa seguir as orientações da VII Diretriz Brasileira de Hipertensão. ${ }^{33}$ A verificação da PA pode ser feita com esfigmomanômetros manuais, semiautomáticos ou automáticos. A recomendação é realizá-la várias vezes com o paciente sentado em ambiente calmo e confortável, para melhorar a reprodutibilidade e aproximar os valores obtidos no consultório àqueles fornecidos pelo MAPA na vigília.

Deve ser observada a possibilidade de ocorrer efeito do avental branco, fenômeno que envolve duas situações. A primeira é a hipertensão do avental branco, quando a PA está elevada em medidas isoladas no consultório e normais no MAPA ou no MRPA. A segunda situação é o efeito do avental branco, caracterizado por PA de consultório elevada em relação à média de pressão de vigília no MAPA ou à média semanal do MRPA, sem haver alteração do diagnóstico, seja de hipertensão ou normotensão. ${ }^{35}$

Essas duas situações podem levar ao falso diagnóstico de HAR, acarretando a realização de exames e o uso de medicação de modo desnecessário. É possível referir-se à hipertensão do avental branco como uma causa de hipertensão arterial pseudorresistente. ${ }^{36}$

\subsection{Monitoramento Ambulatorial da Pressão Arterial em Hipertensão Arterial Resistente}

Esse exame é necessário para afastar a hipótese de hipertensão do avental branco, que falsamente sugere 
HAR. ${ }^{37} \mathrm{O}$ diagnóstico se confirma quando as médias pressóricas nos períodos de vigília e de 24 h estão abaixo de 135/85 mmHg e de 130/80 mmHg, respectivamente. Quando comparados com as medidas casuais da PA, os valores obtidos são mais fortemente relacionados com os riscos decorrentes da hipertensão arterial, principalmente nos exames de MAPA nos quais se identifica uma ausência ou atenuação do descenso da pressão com o sono, como também um aumento no diferencial de pressão sistólica-diastólica. ${ }^{37}$ O Quadro 2 apresenta as principais aplicabilidades do MAPA em hipertensão arterial, exame fundamental na avaliação, no diagnóstico e na evolução da HAR.

\subsection{Monitoramento Residencial da Pressão Arterial e Automedida da Pressão Arterial}

As medidas domiciliares da PA são mais precisas do que a medida casual e apresentam melhor predição de risco para os desfechos $\mathrm{CV}$, contribuindo para maior adesão ao tratamento medicamentoso. ${ }^{35,38,39}$ Nesse contexto, a MRPA e a automedida da PA (AMPA) se apresentam como alternativas viáveis e eficazes tanto para o diagnóstico adequado quanto para a melhora na adesão. ${ }^{40,41}$

\subsection{Medida da Pressão Arterial Central}

A rigidez arterial é reconhecida como um importante índice prognóstico e potencial alvo terapêutico em pacientes hipertensos. Em função disso, a pressão sistólica central (PSc) e a VOP foram investigadas recentemente em uma população de pacientes com HAR. ${ }^{42} \mathrm{~A}$ idade média dessa população era de 58,7 $\pm 15,3$ anos, e $65 \%$ $(n=53)$ eram do sexo feminino. A pressão braquial e central estavam aumentadas em todos os pacientes, e o valor de VOP foi superior ao valor de referência para a idade, sendo essa diferença estatisticamente maior para VOP no sexo feminino.

Outro estudo 23 tratou de observar se existia uma associação entre HAR e rigidez arterial e mostrou que os pacientes com HAR apresentaram maior rigidez vascular do que o grupo com hipertensão bem controlada. A VOP aumentou com a rigidez arterial e foi correlacionada aos níveis de PA, justificando a necessidade de um controle adequado dela.

\section{Quadro 2 - Principais informações obtidas com o monitoramento ambulatorial da pressão arterial}

\footnotetext{
- Múltiplas medidas ao longo de um período de observação

- Avaliação pressórica durante o período de vigília

- Correlação das medidas de vigília com atividades e sintomas

- Avaliação pressórica durante o sono

- Possibilidade de correlação da variabilidade pressórica com sintomas, atividades e fármacos

- Complementação diagnóstica e prognóstica do paciente

- Avaliação do efeito anti-hipertensivo
}

\section{Lesões de Órgãos-Alvo}

Coordenador: Roberto Dischinger Miranda.

Autores: José Fernando Vilela-Martin, Juan Carlos YugarToledo, Wilson Nadruz Júnior e Cibele Isaac Saad Rodrigues.

\subsection{Introdução}

A HAR, controlada ou não, está associada a maior prevalência de lesão de órgãos-alvo (LOA) e a maior risco CV e de mortalidade, quando comparada à hipertensão arterial controlada. ${ }^{43-45}$ Por isso, a investigação de LOA na HAR é fundamental para complementar a estratificação de risco e estabelecer o prognóstico. ${ }^{44}$

\subsection{Alterações Vasculares}

Hipertensos resistentes apresentam alterações vasculares funcionais e estruturais decorrentes não só da hipertensão arterial não controlada, mas também do envelhecimento vascular precoce.. Esse é um processo complexo que envolve alterações bioquímicas, enzimáticas e celulares que modificam a função e a estrutura do vaso, culminando com a degeneração precoce e progressiva da saúde arterial. ${ }^{43-47}$

Os mecanismos fisiopatológicos incluem aumento do estresse oxidativo, disfunção endotelial, remodelamento vascular, hipertrofia de células musculares lisas, aumento da rigidez arterial por alterações na distribuição de colágeno/ elastina, inflamação vascular e maior expressão de mediadores inflamatórios e metaloproteinases de reparação de matriz, além de aumento dos produtos finais da glicação avançada e calcificação parietal. ${ }^{48,49}$

Entre os mecanismos moleculares do envelhecimento vascular, citam-se as alterações genéticas de segmentos envolvidos na proteção e reparação do $\mathrm{DNA}^{50}$ e na atividade metabólica mitocondrial. ${ }^{51}$

$\mathrm{Na}$ microcirculação, a disfunção endotelial promove vasoconstrição, remodelamento eutrófico (aumento da relação média/lúmen [M/L] sem modificação externa), diminuição da reserva vasodilatadora e rarefação vascular, esta última avaliada por capilaroscopia in vivo, ${ }^{52}$ biópsia de glúteo ou, ainda, por mensuração da relação $\mathrm{M} / \mathrm{L}$ com Dopplerfluxometria a laser de artérias retinianas ${ }^{53}$ e videomicroscopia óptica. Nas grandes artérias, o remodelamento parietal leva ao aumento da rigidez arterial. ${ }^{49,54-56}$

A rigidez arterial é estimada pela VOP carótidafemoral (VOP c-f), e o cálculo do índice de incremento (augmentation index (Alx), por tonometria de aplanação. ${ }^{57,58}$ Essas alterações de parâmetros hemodinâmicos e biomarcadores celulares da rigidez arterial estão associadas ao aumento da morbimortalidade. ${ }^{59,60}$

O comprometimento macrovascular é caracterizado ainda pelas doenças aterosclerótica carotídea, cerebral, coronariana e periférica. ${ }^{61,62}$

\subsection{Alterações Cerebrais}

O comprometimento cerebrovascular na HAR ocorre de maneira sutil e insidiosa. Lesões microscópicas da substância branca têm início precoce e podem evoluir de modo 
irreversível, promovendo déficit cognitivo e progressão para demência vascular. ${ }^{63,64}$

Pacientes com HAR têm maior risco de infarto cerebral e isquemia cerebral transitória, fato apontado pelos estudos KAISER Permanente ${ }^{16}$ e REGARDS, ${ }^{65}$ que mostram um aumento de risco de 17 e 14\%, respectivamente. Aterosclerose carotídea e dos pequenos vasos cerebrais são responsáveis por fenômenos isquêmicos e tromboembólicos. A oclusão de artéria retiniana é um marcador de lesão de pequenos vasos e tem sido associada a maior risco de evento cerebral. ${ }^{66}$

Hipertensão não controlada é a principal causa de acidente vascular hemorrágico. Pacientes com HAR apresentam microangiopatia (aneurismas de Charcot-Bouchard), que afetam as artérias penetrantes do cérebro e causam hemorragia intraparenquimatosa. ${ }^{67}$

Alterações na rigidez de grandes artérias também estão associadas a maior ocorrência de alterações microvasculares e maior predisposição para eventos cerebrovasculares. ${ }^{68}$

\subsection{Alterações Cardíacas}

Diversas alterações cardíacas, tais como HVE, disfunção diastólica do ventrículo esquerdo (DDVE) e isquemia miocárdica, podem ser observadas em pacientes com HAR. ${ }^{69}$ A HVE é um preditor independente de insuficiência cardíaca, doença arterial coronária (DAC), arritmias e acidente vascular encefálico. ${ }^{70}$

No Brasil, a prevalência de HVE em pacientes com HAR, avaliada por ecocardiografia, varia de 68 a $87 \%,{ }^{71,72}$ sendo a HVE concêntrica ao padrão geométrico mais encontrado nesses indivíduos. ${ }^{72,73}$

A DDVE predispõe a eventos cardiovasculares e insuficiência cardíaca, independentemente da massa cardíaca e dos níveis de PA. ${ }^{74}$ A prevalência exata de DDVE em pacientes com HAR é incerta, mas a forte associação entre essa condição e a $\mathrm{HVE}^{74}$ sugere que a DDVE é bastante frequente nessa população. Cerca de um terço dos pacientes com HAR têm diagnóstico de DAC. ${ }^{71}$ Contudo, mesmo na ausência de DAC manifesta, até $28 \%$ dos pacientes com HAR apresentam isquemia miocárdica, ${ }^{72}$ a qual pode resultar de diminuições na reserva coronária, de aumentos no consumo de oxigênio miocárdico, especialmente nos portadores de HVE, e de aumentos na rigidez arterial. ${ }^{70,74}$

\subsection{Alterações Renais}

A associação entre HAR e DRC está bem estabelecida, podendo ser causa ou consequência. O substrato anatomopatológico é a nefroesclerose hipertensiva, decorrente de alterações hemodinâmicas (hiperfiltração e hipertrofia glomerular) que culminam em glomeruloesclerose. A nefroesclerose, denominada erroneamente como "benigna", caracteriza-se por arteriosclerose e arteriolosclerose, hialinose, lesões tubulointersticiais e glomerulosclerose segmentar focal e global.

São fatores de risco conhecidos para progressão da DRC: idade > 50 anos, sexo masculino, predisposição genética, história familiar, afrodescendência, duração e estágio da hipertensão arterial, baixo nível socioeconômico, intensidade da albuminúria, grau de disfunção renal, dislipidemia, obesidade, diabetes, estilo de vida (dieta hipersódica, hiperproteica e fumo), uso de substâncias nefrotóxicas, entre outros. ${ }^{75}$ Albuminúria e redução do ritmo de filtração glomerular estimado (RFG-e) identificam pacientes de alto risco $\mathrm{CV}$ e renal, e a diminuição da albuminúria pode ser objetivo terapêutico na HAR. ${ }^{26-28}$

$\mathrm{Na}$ avaliação e no acompanhamento da lesão renal, são recomendados: exame de urina, creatinina sérica para estimar o RFG pelas fórmulas MDRD ou CKD-EPI, disponíveis no site http://ckdepi.org/equations/gfr-calculator/, ultrassonografia renal e de vias urinárias e cálculo da razão albuminúria ou proteinúria/creatininúria visando à classificação do estágio de $\mathrm{DRC}^{75}$ (Figura 2).

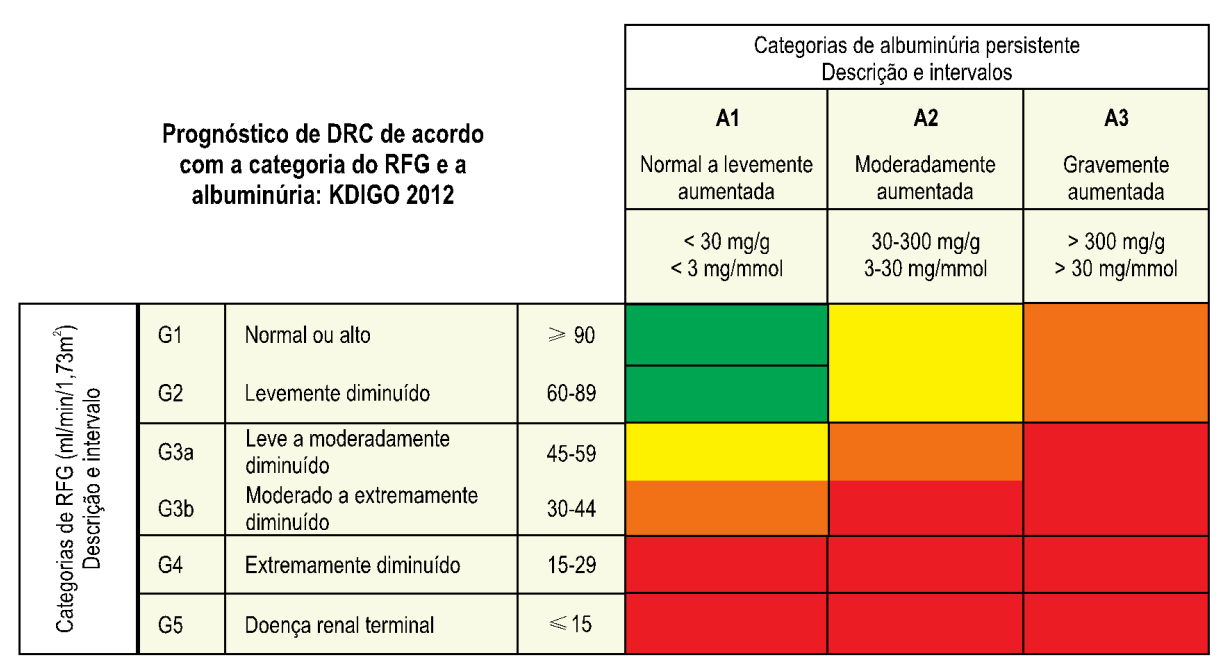

Figura 2 - Prognóstico da doença renal crônica de acordo com os graus de albuminúria e de declínio do RFG-e. ${ }^{76}$ Verde: baixo risco; amarelo: risco moderado; laranja: alto risco; vermelho: risco muito alto. 


\section{Fenótipo do paciente com Hipertensão Arterial Resistente}

Coordenador: Luciano Ferreira Drager

Autores: Heitor Moreno Júnior, Juan Carlos Yugar-Toledo e Luiz Aparecido Bortolotto

\subsection{Introdução}

Nesta seção, serão descritas inicialmente as características que distinguem um hipertenso resistente de um não resistente. Posteriormente, serão discutidas as diferenças entre os hipertensos resistentes controlados e os não controlados, finalizando com a abordagem do fenótipo extremo dos pacientes com hipertensão resistente, que é o hipertenso refratário.

\subsection{Fenótipo do paciente com Hipertensão Arterial Resistente}

O paciente com HAR apresenta comumente algumas características que o distinguem daqueles com a não resistente, tais como: idade mais avançada; obesidade; perfil de alta ingestão de sal; DRC; diabetes; presença de LOA, como a hipertrofia ventricular esquerda; sexo feminino e raça negra. ${ }^{1} \mathrm{O}$ estudo multicêntrico brasileiro ReHOT mostrou que diabetes, história prévia de acidente vascular encefálico e PA na entrada do estudo $\geq 180 / 110$ $\mathrm{mmHg}$ (estágio 3 da hipertensão arterial) foram preditores independentes da verdadeira resistência. ${ }^{13}$ Enquanto algumas dessas características são intuitivas, outras como ser do sexo feminino, ainda não têm explicações bem definidas para a predição na HAR.

\subsection{Fenótipo da Hipertensão Arterial Resistente Controlada e da Não Controlada}

\subsubsection{Aspectos Fisiopatológicos}

A HAR-C evidencia maior dependência do status volêmico que a HAR-NC, devido a importante persistência de retenção hídrica, sensibilidade aumentada ao sódio, hiperaldosteronismo e disfunção renal. Além disso, maior expansão de conteúdo plasmático avaliado por bioimpedância torácica, ${ }^{77}$ maior concentração de aldosterona plasmática e urinária, supressão da atividade de renina, ${ }^{78}$ elevada relação aldosterona/renina plasmática, assim como altos níveis de peptídeo natriurético atrial (ANP) e cerebral (BNP) são observados nesses indivíduos. ${ }^{79-83}$ Essa relação entre volume e pressão elevados é a base fisiopatológica demonstrada em vários estudos ${ }^{81,84,85}$ e justifica o uso de diuréticos em pacientes com HAR-C. ${ }^{86,87}$

Em contraste, portadores de HAR-NC frequentemente têm hiperatividade do sistema simpático, evidenciada por elevação de metanefrinas urinárias $(24$ h) e da frequência cardíaca de repouso e redução da sua variabilidade em 24 h (análise espectral), além de maior rigidez vascular (aumento da VOP). ${ }^{88,89}$ Esses marcadores de atividade simpática aumentada, em conjunto com outros fatores ligados ao hiperaldosteronismo, ${ }^{78,90-92}$ estão vinculados a mecanismos que mantêm a PA alta mesmo com o uso de quatro ou mais agentes anti-hipertensivos, caracterizando a HAR-NC. Valores mais elevados de VOP denotam rigidez arterial exacerbada, ${ }^{4}$ e níveis elevados de citocinas, incluindo o fator de necrose tumoral alfa (TNF- $\alpha$ ), ${ }^{48,56,93}$ provavelmente assinalam o dano vascular em pacientes com HAR. ${ }^{49}$

Outros fatores e mecanismos, como idade, obesidade, AOS, ${ }^{4,94,95}$ afrodescendência, adipocitocinas alteradas, ${ }^{96}$ disfunção endotelial, maior atividade das metaloproteinases-2 e 9 e das moléculas de adesão ${ }^{97-99}$ também estão envolvidos nesse processo.

Polimorfismos genéticos, especialmente do sistema reninaangiotensina-aldosterona e da sintase endotelial do óxido nítrico (eNOS) vêm sendo correlacionados à HAR ${ }^{100,101}$ (Figura 3); todavia, grandes estudos convenientemente caracterizados em indivíduos com a doença são necessários para definir a importância da genética nesse grupo de pacientes.

\subsubsection{Diferenças Clínicas}

Em 2011, Martins et al. publicaram um estudo comparativo entre pacientes com HAR-C e HAR-NC, ${ }^{4}$ mais exatamente sobre fatores biológicos que contribuiriam para a resistência aos anti-hipertensivos. Os índices de massa corporal, rigidez arterial (VOP), índice de massa ventricular esquerda (IMVE) e concentração de aldosterona plasmática (AP) foram maiores no grupo da HAR-NC, quando comparados com o grupo da HAR-C. Além disso, por análise multivariada, os autores demonstraram que a VOP era dependente da idade nos grupos, embora sua influência fosse mais importante nos pacientes com HAR-NC.

Eles também demonstraram que o grupo da HAR-NC apresentava maiores valores de espessura íntima média de carótidas (EIMC) e VOP. ${ }^{102}$ Finalmente, o descenso do sono (dipping pattern) foi menos pronunciado no grupo da HAR-NC. ${ }^{103}$

\subsubsection{Prognóstico}

Pierdomenico et al. ${ }^{104}$ avaliaram desfechos CV em indivíduos com HAR-C e HAR-NC. A ocorrência de eventos CV fatais e não fatais foi investigada em 340 pacientes com HAR-C (PA < 140/90 mmHg ou PA diurna $<135 / 85 \mathrm{mmHg}$ ) e 130 com HAR-NC (PA $\geq 140$ ou $90 \mathrm{mmHg}$ e PA diurna $>135$ ou $85 \mathrm{mmHg}$ ). Durante o seguimento (4,98 $\pm 2,9$ anos), as taxas de eventos por 100 pacientes/ano foram de 0,87 e 4,1, respectivamente. Esses dados mostraram também que pacientes com HAR-NC têm maior risco de DAC, acidente vascular encefálico, arteriopatia, insuficiência cardíaca congestiva (ICC), doença renal e mortes de todas as causas quando comparados a pacientes com HAR-C.

\subsection{Fenótipo do Paciente com Hipertensão Arterial Refratária}

A hipertensão arterial refratária parece ser um fenótipo extremo do hipertenso resistente. Recentemente, a caracterização fenotípica mostrou que esses pacientes são mais jovens do que os resistentes em geral, mais comumente mulheres, com maior frequência de insuficiência cardíaca 


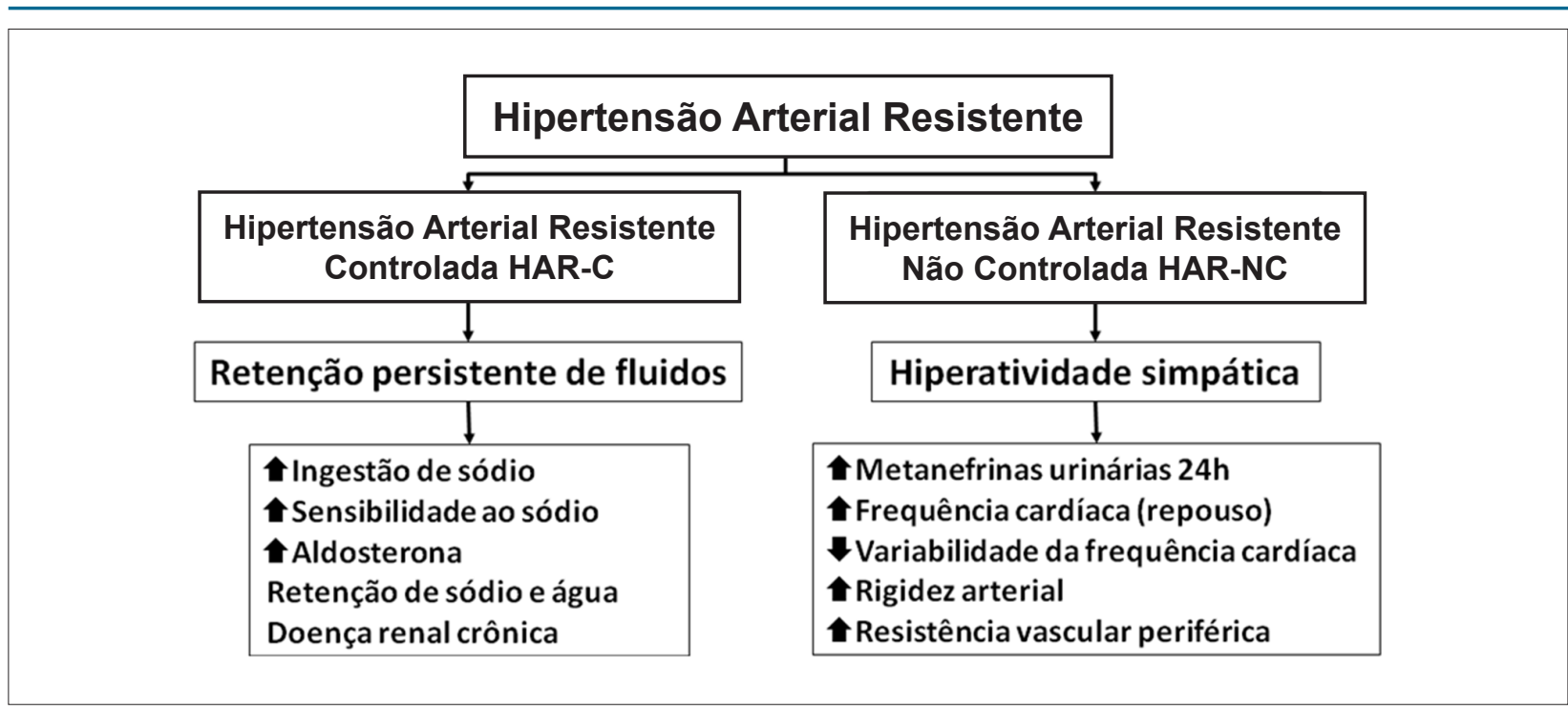

Figura 3 - Mecanismos fisiopatológicos predominantes na hipertensão resistente controlada (HAR-C) e não controlada (HAR-NC). Hipertensão arterial refratária (não controlada com cinco fármacos ou mais) é incluída no grupo da HAR-NC.

e, de maneira destacada, têm maior atividade simpática do que os pacientes resistentes. ${ }^{5}$ Esses achados são importantes pilares para a fisiopatologia da refratariedade, constituindo potencialmente um alvo terapêutico para procedimentos como a desnervação renal. Estudos nesta área estão atualmente em desenvolvimento.

\section{Causas Secundárias de Hipertensão Arterial Resistente}

Coordenadora: Fernanda Marciano Consolim-Colombo

Autores: Márcio Gonçalves de Sousa, Flávio Antonio de Oliveira Borelli, Cibele Isaac Saad Rodrigues e Fernanda Marciano Consolim-Colombo

\subsection{Introdução}

A hipertensão arterial secundária (HASec) é definida como um aumento da PA devido a uma causa identificável. ${ }^{33,105}$ Frente a um paciente com HAR, torna-se imperativa a investigação das causas mais prevalentes de HASec "não endócrina" e "endócrina" após exclusão de uso de fármacos que podem interferir com os valores pressóricos: antiinflamatórios, glicocorticoides, descongestionantes nasais, inibidores de apetite, antidepressivos, imunossupressores, eritropoietina, contraceptivos e drogas ilícitas. ${ }^{33,105}$

\subsection{Hipertensão Arterial Secundária de Causas Não} Endócrinas

\subsubsection{Apneia Obstrutiva do Sono}

Definida como cessação total ou parcial do fluxo respiratório durante o sono, esta síndrome promove dessaturação da oxi-hemoglobina e microdespertares durante o sono. Estima-se que a prevalência de AOS seja de 17\% dos adultos americanos ${ }^{106}$ e $30 \%$ da população de hipertensos, podendo afetar 60 a $80 \%$ dos hipertensos resistentes. ${ }^{94}$ Uma recente metanálise ${ }^{107}$ concluiu que a presença de AOS está relacionada a maior risco de HAR. ${ }^{107}$

A ativação do sistema nervoso simpático e as alterações humorais são responsáveis por modificações na integridade do endotélio vascular, e suas consequências nos pacientes com AOS incluem aumento da PA, desenvolvimento de doença aterosclerótica, arritmias cardíacas, dentre outras. ${ }^{108}$ A suspeita clínica pode ser rastreada a partir do questionário de Berlin. ${ }^{109}$

O diagnóstico é feito com o exame de polissonografia, que registra os índices de apneia/hipopneia superiores a cinco eventos/hora.

No tratamento, devem ser instituídas orientações sobre higiene do sono e perda de peso, dentre outras; para a desobstrução das vias de respiração, o uso de equipamento que produz pressão positiva contínua nas vias respiratórias (CPAP, continuous positive airway pressure) é o mais difundido. No entanto, o impacto desse tratamento na redução dos valores de PA ainda é matéria de debate. ${ }^{110,111}$

\subsubsection{Doença do Parênquima Renal}

A doença do parênquima renal (DPR) é uma das causas mais prevalentes de HASec. Seu diagnóstico é relativamente simples, pois a investigação da função dos rins faz parte da abordagem de rotina do hipertenso. A hipertensão arterial tem alta prevalência nos pacientes em diálise e nos transplantados renais, e os eventos CV são os responsáveis pela alta morbimortalidade dessa população. ${ }^{112}$

A progressão da disfunção renal nos portadores de DPR é diretamente relacionada aos valores pressóricos, e as metas pressóricas devem ser alcançadas para reduzir a morbimortalidade CV. Nos portadores de DPR e nos transplantados renais, os IECA e os antagonistas dos receptores da angiotensina II demonstraram proteção adicional aos rins, 


\section{Posicionamento}

além da obtida pela redução do nível pressórico, sendo, por isso, os fármacos preferenciais. ${ }^{33,105,113}$

\subsubsection{Estenose da Artéria Renal}

A doença renovascular é o termo usado para definir o acometimento das artérias renais por diferentes patologias, como doença aterosclerótica, displasia fibromuscular e vasculites, que podem levar à obstrução dos vasos. Quando há discreta obstrução arterial, comumente não há sintomas associados; porém, quando há obstruções superiores a 70\% da artéria, pode ocorrer hipertensão arterial grave e até mesmo nefropatia isquêmica.

A estenose da artéria renal (EAR) de origem aterosclerótica está presente em 12,5\% dos pacientes hipertensos resistentes com idade acima de 50 anos. ${ }^{114} \mathrm{O}$ diagnóstico deve sempre ser feito, mas o manuseio dessa condição ainda é muito discutido na literatura. ${ }^{115,116} \mathrm{O}$ controle pressórico adequado e o bloqueio na progressiva deterioração da função renal são os objetivos principais do tratamento desses pacientes. Para isso, duas são as possibilidades terapêuticas nesta população: clínica ou intervencionista (cirúrgica ou percutânea, com ou sem implante de próteses vasculares [stents]).

As intervenções são recomendadas para os pacientes com HAR ou hipertensão arterial acelerada com perda progressiva da função renal, com estenose bilateral ou com estenose em rim "único", ou com graves complicações (ICC e edema agudo de pulmão de repetição). ${ }^{33,115,116}$

Outras potenciais indicações cirúrgicas são: obstrução total da artéria renal, grandes fístulas arteriovenosas, lesão de aorta englobando as artérias renais e insucesso no tratamento clínico ou endovascular. ${ }^{117}$

\subsection{Hipertensão Arterial Secundária de Causas Endócrinas}

\subsubsection{Hiperaldosteronismo Primário}

Considerado no passado como um tipo raro de HASec (prevalência na ordem de 1\%), atualmente, julga-se que o hiperaldosteronismo pode chegar até $22 \%$ em populações com HAR. ${ }^{118,119} \mathrm{O}$ adenoma da suprarrenal é a causa mais frequente, sendo a hiperplasia uni ou bilateral menos detectadas. Carcinomas, apesar de infrequentes, ou formas genéticas, também podem ser responsáveis pela instalação da doença.

A aldosterona, por meio da ativação dos receptores mineralocorticoides, está relacionada à resistência insulínica e à disfunção endotelial; consequentemente, ela participa do desenvolvimento da síndrome metabólica e das lesões $\mathrm{CV}$ e renais associadas ao quadro de HAR. Assim, o bloqueio desses receptores mineralocorticoides promove melhora da disfunção endotelial e contribui para melhor resposta ao tratamento da HAR e das LOA. ${ }^{118,119}$

Na realização do diagnóstico, todos os portadores de HAR (não apenas aqueles que apresentem hipocalemia) devem ser avaliados quanto à ocorrência de hiperaldosteronismo. ${ }^{33}$ A triagem inclui avaliação da razão aldosterona plasmática (expressa em $\mathrm{ng} / \mathrm{dL}$ ) pela atividade de renina plasmática (expressa $\mathrm{ng} / \mathrm{mL} / \mathrm{h}$ ) (AP/ARP). Esse método tem grande sensibilidade, mas pode apresentar resultados falso-positivos.
Desse modo, recomenda-se utilizar como valores mínimos de AP e de ARP, respectivamente, $15 \mathrm{ng} / \mathrm{dL}$ e $0,5 \mathrm{ng} / \mathrm{mL} / \mathrm{h}$. Se a razão AP/ARP for $\geq 100$, o diagnóstico será de hiperaldosteronismo; valores < 20 a 30 indicam baixa probabilidade; e valores entre esses extremos detectam "potenciais portadores" dessa condição. ${ }^{120}$ Nesse último caso, testes para avaliação do eixo renina-aldosterona (prova de infusão de volume, caminhada, uso de diuréticos) podem ser realizados.

Para identificação de adenomas ou hiperplasia na suprarrenal por imagem, usa-se a tomografia ou a ressonância magnética. A ausência de um tumor visível à tomografia não exclui um microadenoma, daí a importância da procura de um excesso na produção de aldosterona. Imagens funcionais, obtidas pela cintilografia de adrenal, podem ser úteis na detecção dos adenomas, podendo diferenciá-los das hiperplasias nodulares em até $90 \%$ dos casos. A coleta de amostra de sangue na veia suprarrenal pode ser utilizada para confirmar a lateralização na secreção de aldosterona e a presença de adenoma unilateral. ${ }^{120,121}$

Quanto ao tratamento, na presença de adenoma unilateral, a ressecção unilateral geralmente corrige a produção excessiva de aldosterona e a perda de potássio. A resposta da PA ao tratamento cirúrgico é variável. As hiperplasias são beneficiadas com o bloqueio dos receptores de aldosterona. ${ }^{121}$

\subsubsection{Feocromocitoma}

O feocromocitoma é um tumor neuroendócrino raro, originário de células cromafins (produtoras de catecolaminas), cuja manifestação clínica mais comum é a elevação da PA, podendo ser originário da medula adrenal ou de paragânglios extra-adrenais (paragangliomas). Seu pico de exacerbação clínica está entre a terceira e quarta décadas de vida, mas 10\% dos casos surgem na infância.

O tumor pode apresentar-se de modo esporádico ou associado a síndromes genéticas. ${ }^{122,123}$ Geralmente é unilateral; porém, nas síndromes familiares, pode ser bilateral, múltiplo e extra-adrenal, benigno ou maligno (5 a $26 \%$ dos casos). Essa etiologia deve ser investigada em todos os pacientes que apresentem HAR e/ou sintomas ou sinais sugestivos de liberação adrenérgica. A hipertensão paroxística ocorre em $30 \%$ dos casos, sendo desencadeada por atividades físicas habituais, exercícios mais intensos, procedimentos cirúrgicos e pelo uso de algumas substâncias, como antidepressivos tricíclicos, histamina e opiáceos. Os paroxismos podem ser acompanhados de cefaleia (60 a 90\%), sudorese (55 a 75\%) e palpitações (50 a 70\%). Sintomas de insuficiência cardíaca e alterações no eletrocardiograma podem ser indicativos de miocardite induzida por excesso de catecolaminas.

No diagnóstico, a dosagem de metanefrinas (metabólitos das catecolaminas), tanto no plasma quanto na urina de $24 \mathrm{~h}$, apresenta maior sensibilidade e especificidade que a dosagem direta de catecolaminas. Quando os exames laboratoriais não forem elucidativos, o teste de supressão com clonidina pode ser realizado (administração de 0,2 mg de clonidina com dosagem de catecolaminas $1 \mathrm{~h}$ antes e 2 h após a ingestão do fármaco).

Para o diagnóstico topográfico dos tumores e eventualmente de metástases, os métodos de imagens recomendados são 
tomografia computadorizada e ressonância magnética, ambas com sensibilidade próxima a $100 \%$ para tumores adrenais. O mapeamento de corpo inteiro com metaiodobenzilguanidina (MIBG) 131 ou 121 apresenta sensibilidade de 56 a 85\% (tumores malignos) e alta especificidade. O octreoscan, o mapeamento ósseo e o PET scan (com diferentes marcadores) podem ser decisivos quando os exames de localização anteriores são negativos ou na investigação de doença maligna.

O tratamento é cirúrgico. Porém, na terapia medicamentosa pré-operatória ou crônica, são usados inicialmente alfabloqueadores (prazosin, doxazocin e dibenzilina), combinados ou não a outros agentes, como betabloqueadores (após alfabloqueio efetivo), IECA e BCC. Para a intervenção cirúrgica, recomenda-se controle prévio dos níveis de PA e reposição volêmica. ${ }^{124} \mathrm{Em}$ crises agudas e durante a cirurgia, nitroprussiato de sódio pode ser utilizado. ${ }^{124}$

\subsubsection{Hipotireoidismo e Hipertireoidismo}

A hipertensão arterial pode estar presente em $40 \%$ dos portadores de distúrbios da tireoide, pois a correção da disfunção glandular geralmente é responsável pelo controle da PA. ${ }^{125}$ Uma vez corrigido o hipo ou o hipertireoidismo, e persistindo níveis elevados de PA, está indicado o uso de fármacos anti-hipertensivos. ${ }^{32,126}$

As causas de HASec em pacientes com HAR são sumarizadas na Tabela 1.

Tabela 1 - Prevalência, achados clínicos e estudos adicionais das causas mais comuns de hipertensão arterial secundária em pacientes com hipertensão arterial resistente

\begin{tabular}{|c|c|c|c|c|}
\hline Causa secundária & Prevalência geral & Prevalência na HAR & Achados clínicos & Investigação diagnóstica \\
\hline $\begin{array}{l}\text { Apneia obstrutiva do } \\
\text { sono }^{94,107,109}\end{array}$ & $>5$ a $15 \%$ & $>30 \%$ & $\begin{array}{l}\text { Ronco, sonolência diurna, cefaleia } \\
\text { matinal, síndrome metabólica }\end{array}$ & $\begin{array}{l}\text { Questionário de Berlim, stop-bang, Escala de } \\
\text { sonolência de Epworth } \\
\text { Polissonografia (padrão-ouro) ou polissonografia } \\
\text { residencial. Diagnóstico. Índice apneia e/ou hipopneia } \\
>5 \text { eventos por hora de sono }\end{array}$ \\
\hline $\begin{array}{l}\text { Doença do } \\
\text { parênquima renal }{ }^{113}\end{array}$ & 1,6 a $8 \%$ & 2 a $10 \%$ & $\begin{array}{l}\text { Edema, anorexia, nictúria, fadiga, } \\
\text { anemia, ureia e creatinina elevadas, } \\
\text { alterações do sedimento urinário }\end{array}$ & $\begin{array}{l}\text { Exame de urina (densidade baixa, hematúria } \\
\text { glomerular ou albuminúria), cálculo do RFG estimado, } \\
\text { US renal, Pesquisa de albuminúria e relação } \\
\text { proteinúria/creatininúria em amostra isolada }\end{array}$ \\
\hline $\begin{array}{l}\text { Estenose da artéria } \\
\text { renal }^{115,116}\end{array}$ & 1 a $8 \%$ & 2,5 a $20 \%$ & $\begin{array}{l}\text { Sopro abdominal, edema agudo de } \\
\text { pulmão, alteração da função renal } \\
\text { por medicamentos que bloqueiam o } \\
\text { SRAA, rins assimétricos }\end{array}$ & $\begin{array}{c}\text { Rastreio: US com Doppler de artérias renais } \\
\text { (operador dependente) e/ou renograma com ou } \\
\text { sem captopril, angiorressonância, tomografia } \\
\text { computadorizada, arteriografia renal convencional } \\
\text { (padrão-ouro) }\end{array}$ \\
\hline $\begin{array}{l}\text { Doenças da tireoide }{ }^{32} \\
\text { Hipotiroidismo }\end{array}$ & & & $\begin{array}{l}\text { Fadiga, ganho de peso, perda de } \\
\text { cabelo, hipertensão arterial sistólica, } \\
\text { fraqueza muscular. }\end{array}$ & \\
\hline Hipertiroidismo & 1 a $2 \%$ & 1 a $3 \%$ & $\begin{array}{l}\text { Intolerância ao calor, perda de } \\
\text { peso, hipertensão arterial diastólica, } \\
\text { palpitações, exoftalmia, tremores, } \\
\text { taquicardia }\end{array}$ & TSH e T4 Livre \\
\hline $\begin{array}{l}\text { Síndrome de } \\
\text { Cushing }^{32}\end{array}$ & $0,5 \%$ & $<1 \%$ & $\begin{array}{l}\text { Ganho de peso, fadiga, hirsutismo, } \\
\text { amenorreia, "fácies em lua cheia", } \\
\text { "corcova dorsal", estrias purpúricas, } \\
\text { obesidade central, hipopotassemia }\end{array}$ & $\begin{array}{c}\text { Cortisol salivar } \\
\text { Cortisol urinário de } 24 \text { horas } \\
\text { Cortisol matinal ( } 8 \text { horas) e } 8 \text { horas após } \\
\text { administração de dexametasona ( } 1 \mathrm{mg}) \text { às } 24 \text { h. } \\
\text { Ressonância magnética }\end{array}$ \\
\hline Feocromocitoma $^{127,128}$ & 0,2 a $0,5 \%$ & $<1 \%$ & $\begin{array}{l}\text { Hipertensão arterial episódica, lábil } \\
\text { ou resistente, paroxismos de cefaleia, } \\
\text { sudorese profusa e palpitações, } \\
\text { palidez }\end{array}$ & $\begin{array}{l}\text { Metanefrinas plasmáticas livres e/ou urinárias de } 24 \mathrm{~h} \\
\text { (valores o dobro ou triplo do normal), catecolaminas } \\
\text { plasmáticas e/ou urinárias de } 24 \mathrm{~h} \text { e/ou tomografia } \\
\text { computadorizada e ressonância magnética }\end{array}$ \\
\hline $\begin{array}{l}\text { Coarctação de } \\
\text { aorta }^{129}\end{array}$ & $<1 \%$ & $<1 \%$ & $\begin{array}{c}\text { Diferença de PAS/PAD > 20/10 } \\
\text { mmHg entre membros superiores e } \\
\text { inferiores; sopro ejetivo em região } \\
\text { interescapular }\end{array}$ & $\begin{array}{c}\text { Entalhe da borda inferior da costela na radiografia } \\
\text { de tórax, rastreio com ecodopplercardiograma, } \\
\text { ressonância magnética ou angiografia da aorta } \\
\text { torácica }\end{array}$ \\
\hline
\end{tabular}

Adaptada de Rimoldi SF et al. ${ }^{105}$ AP/ARP: aldosterona plasmática/atividade de renina plasmática; HAR: hipertensão arterial resistente; PAD: pressão arterial diastólica; PAS: pressão arterial sistólica; RFG: ritmo de filtração glomerular; SRAA: sistema renina-angiotensina-aldosterona; US: ultrassonografia. 


\section{Tratamento Não Farmacológico}

Coordenador: Sérgio Emanuel Kaiser

Autores: Gil Fernando Salles, Maria de Fátima de Azevedo e Lucélia Batista Neves Cunha Magalhães.

\subsection{Perda Ponderal}

Vários mecanismos favorecem a manutenção de uma PA elevada em hipertensos obesos, como AOS, hiperatividade simpática, disfunção endotelial, modificação da microbiota intestinal, todos capazes de promover um fenótipo inflamatório e perpetuar um ciclo vicioso. ${ }^{130}$ Pacientes com índice de massa corporal (IMC) $\geq 30 \mathrm{~kg} / \mathrm{m}^{2}$ têm $50 \%$ mais chances de apresentar PA não controlada do que aqueles com IMC normal $\left(<25 \mathrm{~kg} / \mathrm{m}^{2}\right){ }^{131}$ Um IMC > $40 \mathrm{~kg} / \mathrm{m}^{2}$ triplica as chances de se requererem múltiplos fármacos para controle da PA. ${ }^{132}$

Uma perda ponderal de $10 \mathrm{~kg}$ associa-se a uma redução média de 6,0 mmHg na PA sistólica e 4,0 mmHg na PA diastólica. ${ }^{133}$ Surpreendentemente, não há evidências consistentes sobre o efeito da perda de peso induzida por dieta em hipertensos resistentes, mas essa recomendação atende ao bom senso e às evidências disponíveis nos demais subgrupos. Também não existem dados sobre o efeito da cirurgia bariátrica sobre a PA nesse subgrupo. Recente estudo aleatorizado demonstrou redução de ao menos 30\% no número de anti-hipertensivos em 84\% dos pacientes operados, em comparação a $12,4 \%$ do grupo tratado clinicamente. ${ }^{134}$

\subsection{Restrição de Sal}

O controle no consumo de sal é especialmente eficaz em idosos, afrodescendentes e indivíduos com filtração glomerular diminuída. ${ }^{135}$ Nessas situações, restringe-se a capacidade de excreção de água e sódio pelos rins, tornando a PA mais dependente de variações volêmicas. Não por acaso, a sensibilidade ao sódio e a sobrecarga volêmica respondem pelo principal mecanismo fisiopatológico da maioria dos casos de HAR. ${ }^{136}$ Uma revisão sistemática e metanálise envolvendo 34 estudos com 3.230 participantes, sobre o efeito da redução em longo prazo na ingestão de sódio, revelou queda na PA sistólica de 5,8 mmHg (2,5 a 9,2; p = 0,001) associada a redução da excreção urinária de sódio até 100 mmol em 24 h, o que corresponde a uma diminuição da ingestão de sal de aproximadamente $6 \mathrm{~g} / \mathrm{dia} .{ }^{137} \mathrm{Em}$ hipertensos resistentes, uma dieta hipossódica com 2,5 g diários de sal foi capaz de reduzir a PA em até 23,0/9,0 mmHg, em clara demonstração da eficácia dessa medida, não obstante a possibilidade de comprometimento da aderência a longo prazo a tão acentuada restrição no consumo de sal. ${ }^{79}$

\subsection{Ingestão de Álcool}

Fruto da relação direta entre a quantidade de álcool consumida e os níveis pressóricos, o consumo excessivo de álcool contribui significativamente para a dificuldade no controle da PA; $;^{138}$ afinal, o consumo diário de mais de dois "drinks" (cerca de $24 \mathrm{~g} /$ dia) associa-se à elevação dos níveis pressóricos. ${ }^{139}$ Recente metanálise de 36 estudos com 2.865 participantes revelou que a redução de $50 \%$ na ingestão diária de álcool entre os consumidores de seis ou mais "drinks"
(72 g) promoveu queda de 5,50 $\mathrm{mmHg}$ na PA sistólica (IC 95\%; 6,70 a 4,30) e de 3,97 mmHg na PA diastólica (IC 95\%; 4,70 a 3,25). ${ }^{140}$ Não há estudos publicados em hipertensos resistentes; porém, com base nas informações disponíveis, recomenda-se a restrição do consumo diário de álcool a menos de dois "drinks-padrão" (cerca de 24 g) ou até sua cessação.

\subsection{Atividade Física}

Apesar de avaliada apenas em pequenos grupos de hipertensos resistentes, a atividade física é provavelmente tão ou mais benéfica nestes do que em não resistentes. ${ }^{40,141}$ Exercício aeróbico regular diminui a PA de consultório e a ambulatorial em hipertensos resistentes, ${ }^{142-145}$ além de atenuar a característica ativação neuro-humoral. ${ }^{146}$ Não obstante a inexistência de estudos sobre exercício resistido nesse subgrupo, supõe-se haver vantagem ao menos semelhante à observada em hipertensos não resistentes. ${ }^{147}$ Além disso, a melhor capacidade cardiorrespiratória obtida com atividade física parece reduzir a mortalidade de hipertensos resistentes. ${ }^{148}$ Portanto, essa categoria de pacientes deve ser incentivada a realizar atividade física regular de moderada intensidade sob supervisão adequada. Naqueles com PA muito elevada (PA sistólica $\geq 180 \mathrm{mmHg}$ ou PA diastólica $\geq 110 \mathrm{mmHg}$ ), a atividade física deve ser adiada até que a otimização do tratamento medicamentoso promova a redução da PA. ${ }^{40,141}$

\section{Tratamento Farmacológico da Hipertensão Arterial Resistente

\author{
Coordenador: Rui Manoel dos Santos Póvoa
}

Autores: Marcus Vinícius Bolívar Malachias, Armando da Rocha Nogueira e Paulo César Brandão Veiga Jardim

O objetivo do tratamento medicamentoso na HAR é detectar as causas do não controle e encontrar a melhor combinação de fármacos, visando o alcance das metas pressóricas com menor ocorrência de efeitos adversos e maior adesão.

Em geral, busca-se otimizar o tratamento tríplice com os fármacos preferenciais, que são: IECA ou BRA, BCC dihidropiridínico e DT. ${ }^{33,149}$

Os IECA ou BRA, por serem mais bem tolerados, precisam ser elevados às doses máximas na HAR. Deve ser utilizado um DT de longa ação e maior potência, como a clortalidona em lugar da hidroclorotiazida, em doses adequadas ao controle da volemia, de 12,5 a 50 mg, em dose única pela manhã. 1,33,40,150 A indapamida constitui uma segunda opção de DT na HAR. ${ }^{150}$ A furosemida deve ser utilizada em casos de DRC, com RFG igual ou inferior a $30 \mathrm{ml} / \mathrm{min}$. ${ }^{1,33} \mathrm{Na}$ HAR, o BCC deve ser preferencialmente, tomado à noite, para que haja alternância de picos de ação dos anti-hipertensivos. ${ }^{40}$

A intolerância aos BCC, devido a efeitos colaterais, muitas vezes é uma das causas de resistência ao tratamento. Nesses casos, pode ser tentada a utilização de BCCs lipofílicos (manidipino, lercanidipino, manidipino) ou o levanlodipino, 
em baixas doses, ou, em casos selecionados, um BCC não di-hidropiridínico, como diltiazem e verapamil. ${ }^{33} \mathrm{Na}$ impossibilidade de uso de um BCC, pode ser considerada a introdução de um betabloqueador, preferencialmente com ação vasodilatadora, como nebivolol ou carvedilol. ${ }^{33,151}$ Betabloqueadores também podem ser considerados em associação a um ou mais anti-hipertensivos preferenciais - IECA ou BRA, DT, BCC - em condições especiais como insuficiência cardíaca, coronariopatia, frequência cardíaca basal elevada, entre outras. ${ }^{33,150,151}$

O não alcance da meta pressórica com o esquema tríplice exige a utilização de um $4^{\circ}$ fármaco, cuja opção preferencial atual é a espironolactona, de 25 a 50 mg ao dia. ${ }^{13,152-154} \mathrm{Em}$ casos de intolerância à espironolactona, cujo efeito adverso principal é a ocorrência de ginecomastia em homens, pode ser tentada a utilização de 12,5 mg ao dia. Como não há disponibilidade de eplerenone em nosso meio, caso persista a intolerância à espironolactona, mesmo em baixas doses, deverá ser avaliada a sua substituição por um simpatolítico central, preferencialmente a clonidina, de 0,100 a 0,200 mg, duas vezes ao dia, ${ }^{152}$ ou um diurético poupador de potássio, preferencialmente a amilorida (só disponível em nosso meio de forma isolada em formulações magistrais), de 10 a $20 \mathrm{mg} ;{ }^{155}$ ou um betabloqueador, preferencialmente com ação vasodilatadora, se não tiver sido ainda empregado; ${ }^{40}$ ou um alfa bloqueador, preferencialmente a doxazosina, em dosagem de $1 \mathrm{a} 16 \mathrm{mg}$, em uma tomada (noturna) ou duas tomadas diárias. ${ }^{33,40,155}$

Todos esses anti-hipertensivos podem ser utilizados em associações, quando necessário, para o controle pressórico. ${ }^{33}$ Caso não se obtenha o controle com a adição do 4ํo fármaco ou com combinações das opções subsequentes, deve- se utilizar um vasodilatador direto, preferencialmente a hidralazina, em doses diárias de 50 a 150 mg, fracionadas em 2 a 3 tomadas. ${ }^{40} \mathrm{O}$ vasodilatador minoxidil, em face de seus frequentes efeitos adversos, deve ser reservado para situações muito resistentes, quando há falha de todas as alternativas anteriores ${ }^{40,150}$ (Figura 4).

No tratamento da HAR, deve-se estar atento aos possíveis efeitos adversos de cada um dos fármacos empregados, assim como às suas possíveis interações medicamentosas.

\section{Novos Tratamentos da Hipertensão} Arterial Resistente

Coordenador: Luiz Aparecido Bortolotto

Autores: Luiz Aparecido Bortolotto, Luciano Ferreira Drager e Thiago de Souza Veiga Jardim

\subsection{Introdução}

Nos últimos anos, novas formas de tratamento intervencionista têm sido avaliadas para pacientes com HAR, tais como:

\subsection{Estimulação Direta do Seio Carotídeo}

O estímulo dos barorreceptores carotídeos leva ao aumento da atividade dos mesmos e, consequente, redução do fluxo simpático, resultando em diminuição da PA. ${ }^{156}$ Intervenções que promovem esta estimulação têm sido usadas para o tratamento de pacientes com HAR não responsivos a tratamento clínico. ${ }^{156-159}$ A terapia de ativação do barorreflexo (TAB) é um procedimento cirúrgico onde eletrodos são implantados cirurgicamente na porção

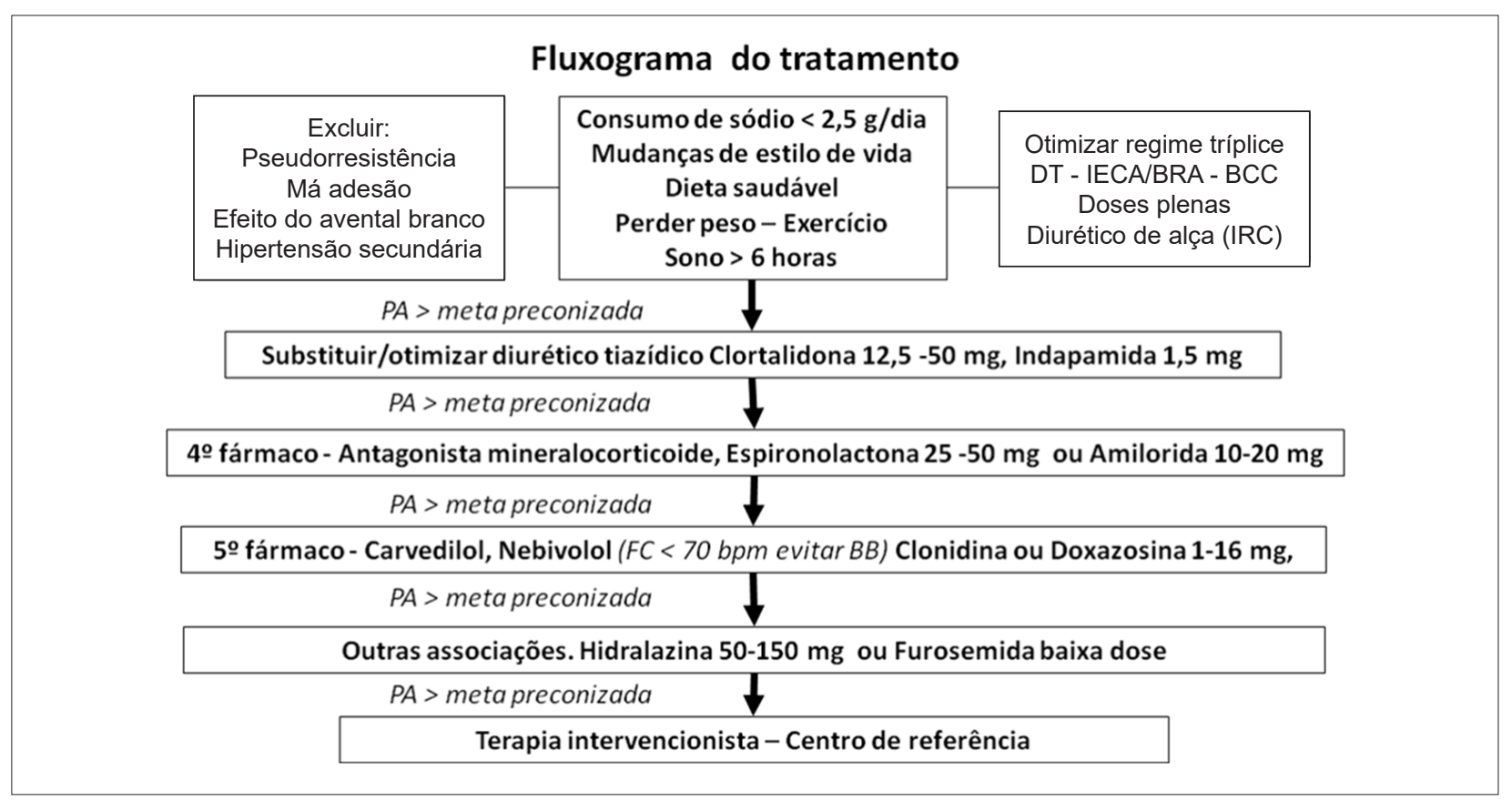

Figura 4 - Fluxograma de tratamento de HA. BCC: bloqueador dos canais de cálcio; BRA: bloqueador do receptor de angiotensina; FC: frequência cardíaca; IECA: inibidor da enzima conversora da angiotensina; IRC: insuficiência renal crônica; PA: pressão arterial. 
externa do seio carotídeo bilateral ou unilateral. ${ }^{157,159}$ A TAB mostrou reduções significativas da PA, que persistem por até 3 anos em estudos randomizados e controlado. ${ }^{157,159}$ Entretanto, o procedimento é invasivo, de alto custo e apresenta efeitos colaterais, que restringem sua indicação na prática clínica. ${ }^{156,159}$ Outra forma de estímulo é a amplificação do barorreflexo endovascular (implante de dispositivo expansível dentro da carótida), que demonstrou resultados promissores no controle da PA na HAR, com maior segurança. ${ }^{156}$ Estes procedimentos não estão disponíveis no Brasil.

\subsection{Denervação Simpática Renal}

A denervação simpática renal (DSR) por cateter de ablação reduz a atividade eferente renal, com consequente aumento do fluxo sanguíneo renal, diminuição da ativação do sistema renina-angiotensina-aldosterona e da retenção de água, e também da atividade aferente renal que, através de sinais cerebrais, diminui ação simpática sobre coração e vasos. ${ }^{160}$

Dados obtidos em estudos não controlados mostraram reduções de até $30 \mathrm{mmHg}$ na PA sistólica de consultório em pacientes com HAR, sem complicações do procedimento. ${ }^{161}$ Entretanto, o estudo SYMPLICITY HTN-3, ${ }^{162}$ randomizado e controlado com procedimento sham, não mostrou efeito significativamente superior de redução da PA após 6 meses com a DSR. Uma metanálise com 11 estudos controlados comparando DSR com tratamento medicamentoso otimizado ou procedimento sham em pacientes com HAR não demonstrou superioridade da DSR em reduzir a PA, havendo heterogeneidade de respostas nos estudos devido principalmente a falta de controle sham na maioria das publicações e heterogeneidade na avaliação da adesão ao tratamento. ${ }^{163}$

O desenvolvimento de novos cateteres circunferenciais com aplicações distais nas artérias renais pode promover DSR mais completa, e efeitos de redução da PA têm sido demonstrados em pacientes hipertensos não tratados. ${ }^{164}$

No posicionamento de 2018 da Sociedade Europeia de Hipertensão, DSR não é recomendada em geral para o tratamento de hipertensão arterial, mas há recomendações para a sua realização no contexto de estudos clínicos controlados com procedimento sham e terapia otimizada para avaliação da segurança e da eficácia em população com grande número de indivíduos. ${ }^{160}$
Com base nessas evidências, no momento, a DSR seria uma alternativa apenas para pacientes com HAR-NC com tratamento farmacológico otimizado e comprovada adesão terapêutica ou com importantes efeitos adversos das medicações, sempre em centros de referência treinados para o procedimento. ${ }^{164}$

\subsection{Uso de Pressão Positiva Contínua em Vias Aéreas}

A AOS é uma condição clínica presente em mais da metade dos pacientes com $\mathrm{HAR}^{94}$ e seu principal tratamento é o CPAP, um compressor de ar que provoca pressão positiva contínua na via aérea do paciente. Até o momento, sete estudos randomizados analisaram o efeito do tratamento da AOS com CPAP em pacientes com HAR. ${ }^{165-171}$ Com exceção de um deles, ${ }^{170}$ os demais encontraram reduções significativas de PA ( 5 mmHg em média; um dos estudos mostrou reduções $\geq 10 \mathrm{mmHg}$ após uso do CPAP). ${ }^{169}$

No entanto, a proporção de pacientes que alcançou a meta de PA ( $<140 / 90 \mathrm{mmHg}$ ) com o CPAP é baixa, possivelmente explicada pela pouca adesão ao CPAP. Na prática clínica observa-se que a resposta da PA ao CPAP pode ser variável, mesmo em pacientes com boa adesão. Recente estudo mostrou biomarcadores preditores de melhor resposta da PA ao uso do CPAP em pacientes com HAR. ${ }^{172}$ A validação e a aplicação em larga escala desses biomarcadores podem ajudar a selecionar melhor os pacientes que terão mais benefícios com a redução da PA.

\subsection{Fístula Arteriovenosa}

A aplicação de uma fístula arteriovenosa (FAV) pode promover diminuição da PA por mecanismos relacionados a: redução na resistência periférica total e no volume sanguíneo, inibição de barorreflexo e liberação de peptídeos natriuréticos. ${ }^{173} \mathrm{Em}$ estudo prospectivo randomizado e controlado, a criação de FAV ilíaca-central por um dispositivo implantável em 44 pacientes com HAR foi acompanhada de significativa redução da PA sistólica de consultório e ambulatorial de $24 \mathrm{~h}$, quando comparada a tratamento medicamentoso. ${ }^{174}$ Entretanto, houve uma taxa elevada de complicações devido a estenose venosa ipsilateral, necessitando de intervenção no grupo com a FAV.

Novos estudos com maior número de pacientes e comparações da FAV com o procedimento sham estão sendo realizados para comprovar os benefícios dela na HAR. ${ }^{173}$ 


\section{Referências}

1. Calhoun DA, Jones D, Textor S, Goff DC, Murphy TP, Toto RD, et al. Resistant hypertension: diagnosis, evaluation, and treatment. A scientific statement from the American Heart Association Professional Education Committee of the Council for High Blood Pressure Research. Hypertension. 2008;51(6):1403-19.

2. Modolo R, de Faria AP, Almeida A, Moreno H. Resistant or refractory hypertension: are they different? Curr Hypertens Rep. 2014;16(10):485.

3. Modolo R, de Faria AP, Sabbatini AR, Moreno H. Resistant hypertension revisited: definition and true prevalence. J Hypertens. 2014;32(7):1546.

4. Martins LC, Figueiredo VN, Quinaglia T, Boer-Martins L, Yugar-Toledo JC, Martin JF, et al. Characteristics of resistant hypertension: ageing, body mass index, hyperaldosteronism, cardiac hypertrophy and vascular stiffness. J Hum Hypertens. 2011;25(9):532-8.

5. Dudenbostel T, Siddiqui M, Oparil S, Calhoun DA. Refractory Hypertension: A Novel Phenotype of Antihypertensive Treatment Failure. Hypertension. 2016;67(6):1085-92.

6. Moreno $\mathrm{H}, \mathrm{Jr}$, Coca A. Resistant and refractory hypertension: reflections on pathophysiology and terminology. Blood Press. 2012;21(4):209-10.

7. Yugar-Toledo JC, Brunelli V, Vilela-Martin JF, Fattori A, Moreno H. Controlled Versus Uncontrolled Resistant Hypertension: Are They in the Same Bag? Curr Hypertens Rep. 2018;20(3):1-6.

8. Mills KT, Bundy JD, Kelly TN, Reed JE, Kearney PM, Reynolds K, et al. Global Disparities of Hypertension Prevalence and Control: A Systematic Analysis of Population-Based Studies From 90 Countries. Circulation. 2016;134(6):441-50.

9. Scala LC, Magalhães LB , Machado A. Epidemiologia da hipertensão arterial sistêmica. In: Sociedade Brasileira de Cardiologia. Livro texto da SBC. 2a ed. São Paulo:Manole;2015. São Paulo; 2015. p. 780-5.

10. Nobre F, Ribeiro AB, Mion D Jr. [Control of arterial pressure in patients undergoing anti-hypertensive treatment in Brazil: Controlar Brazil]. Arq Bras Cardiol. 2010;94(5):663-70.

11. Achelrod D, Wenzel U, Frey S. Systematic review and meta-analysis of the prevalence of resistant hypertension in treated hypertensive populations. Am J Hypertens. 2015;28(3):355-61.

12. Bangalore S, Davis BR, Cushman WC, Pressel SL, Muntner PM, Calhoun DA etal. Treatment-Resistant Hypertension and Outcomes Based on Randomized Treatment Group in ALLHAT. Am J Med. 2017;130(4):439-48 e9.

13. Krieger EM, Drager LF, Giorgi DMA, Pereira AC, Barreto-FilhoJAS, NogueiraAR, et al. Spironolactone Versus Clonidine as a Fourth-Drug Therapy for Resistant Hypertension: The ReHOT Randomized Study (Resistant Hypertension Optimal Treatment). Hypertension. 2018;71(4):681-90.

14. Daugherty SL, Powers JD, Magid DJ, Tavel HM, Masoudi FA, Margolis KL, et al. Incidence and prognosis of resistant hypertension in hypertensive patients. Circulation. 2012;125(13):1635-42

15. Sarafidis PA, Bakris GL. Resistant hypertension: an overview of evaluation and treatment. J Am Coll Cardiol. 2008;52(22):1749-57.

16. Sim JJ, BhandariSK, Shi J, Liu IL, Calhoun DA, McGlynn EA, etal. Characteristics of resistant hypertension in a large, ethnically diverse hypertension population of an integrated health system. Mayo Clin Proc. 2013;88(10):1099-107.

17. Cai A, Calhoun DA. Resistant Hypertension: An Update of Experimental and Clinical Findings. Hypertension. 2017;70(1):5-9.

18. Lazaridis AA, Sarafidis PA, Ruilope LM. Ambulatory Blood Pressure Monitoring in the Diagnosis, Prognosis, and Management of Resistant Hypertension: Still a Matter of our Resistance? Curr Hypertens Rep. 2015;17(10):78

19. Salles GF, Cardoso CR, Muxfeldt ES. Prognostic influence of office and ambulatory blood pressures in resistant hypertension. Arch Intern Med. 2008;168(21):2340-6.
20. MuxfeldtES, Cardoso CR, Salles GF. Prognostic value of nocturnal blood pressure reduction in resistant hypertension. Arch Intern Med. 2009;169(9):874-80.

21. Roush GC, Fagard RH, Salles GF, Pierdomenico SD, Reboldi G, Verdecchia $\mathrm{P}$, et al. Prognostic impact from clinic, daytime, and night-time systolic blood pressure in nine cohorts of 13,844 patients with hypertension. J Hypertens. 2014;32(12):2332-40; discussion 40

22. Laurent S, Boutouyrie P, Asmar R, Gautier I, Laloux B, Guize L, et al. Aortic stiffness is an independent predictor of all-cause and cardiovascular mortality in hypertensive patients. Hypertension. 2001;37(5):1236-41.

23. Chung CM, Cheng HW, Chang J, Lin YS, Hsiao JF, Chang ST, et al. Relationship between resistant hypertension and arterial stiffness assessed by brachial-ankle pulse wave velocity in the older patient. Clin Interv Aging. 2014 Sep 5;9:1495-502

24. Brandao AA, Amodeo C, Alcantara C, Barbosa E, Nobre F, Pinto F, et al. Luso-Brazilian Positioning on Central Arterial Pressure. Arq Bras Cardiol. 2017;108(2):100-8.

25. Salles GF, Cardoso CR, Fiszman R, Muxfeldt ES. Prognostic impact of baseline and serial changes in electrocardiographic left ventricular hypertrophy in resistant hypertension. Am Heart J. 2010;159(5):833-40.

26. Salles GF, Cardoso CR, Fiszman R, Muxfeldt ES. Prognostic importance o baseline and serial changes in microalbuminuria in patients with resistant hypertension. Atherosclerosis. 2011;216(1):199-204.

27. da Costa PM, Cortez AF, de Souza F, Mares GS, Dos Santos BDM, Muxfeld ES. Prognostic impact of baseline urinary albumin excretion rate in patients with resistant hypertension: a prospective cohort study. J Hum Hypertens. 2018;32(2):139-49.

28. Oliveras A, Armario P, Sierra C, Arroyo JA, Hernandez-del-Rey R, Vazquez S, et al. Urinary albumin excretion at follow-up predicts cardiovascular outcomes in subjects with resistant hypertension. Am J Hypertens. 2013;26(9):1148-54.

29. Cortez AF, MuxfeldtES, Cardoso CR, Salles GF. Prognostic Value of C-Reactive Protein in Resistant Hypertension. Am J Hypertens. 2016;29(8):992-1000.

30. de la Sierra A, Segura J, Banegas JR, Gorostidi M, de la Cruz JJ, Armario P, et al. Clinical features of 8295 patients with resistant hypertension classified on the basis of ambulatory blood pressure monitoring. Hypertension. 2011;57(5):898-902.

31. White WB. Ambulatory blood pressure monitoring as an investigative tool for characterizing resistanthypertension and its rational treatment. J Clin Hypertens (Greenwich). 2007;9(1 Suppl 1):25-30

32. Williams B, Mancia G, Spiering W, Agabiti Rosei E, Azizi M, Burnier M, et al 2018 ESC/ESH Guidelines for the management of arterial hypertension. Eur Heart J. 2018;39(33):3021-104

33. Malachias M, PlavnikFL, MachadoCA, MaltaD, ScalaLCN, Fuchs S, 7th Brazilian Guideline of Arterial Hypertension: Chapter 1 - Concept, Epidemiology and Primary Prevention. Arq Bras Cardiol. 2016;107(3 Suppl 3):1-6.

34. Mancia G, Fagard R. Guidelines for the management of hypertension and target organ damage: reply. J Hypertens. 2013; 31(12):2464-5

35. Nobre FMJ, Gomes MAM, Barbosa ECD, Rodrigues CIS, Neves MFT et al. $6^{a}$ Diretrizes de Monitorização Ambulatorial da Pressão Arterial e 4a - Diretrizes de Monitorização Residencial da Pressão Arterial. Arq Bras Cardiol 2018; 2018;110(5 Supl.1):1-29.

36. Silva GV, Mion D. Papel da MAPA e da MRPA na avaliação de pacientes com hipertensão de difícil controle. Rev Bras Hipertens. 2008;15(1):17-20.

37. Muxfeldt ES, Bloch KV, Nogueira AR, Salles GF. Twenty-four hour ambulatory blood pressure monitoring pattern of resistanthypertension. Blood Press Monit. 2003;8(5):181-5.

38. Souza WKS, Jardim PCB, Porto LB, Araújo FA, Sousa ALL, Salgado CM Comparação e correlação entre automedida, medida casual e monitorização ambulatorial da pressão arterial. Arq Bras Cardiol. 2011:97(2):148-55. 
39. Breaux-Shropshire TL, Judd E, Vucovich LA, Shropshire TS, Singh S. Does home blood pressure monitoring improve patient outcomes? A systematic review comparing home and ambulatory blood pressure monitoring on blood pressure control and patient outcomes. Integr Blood Press Control. 2015;8:43-9.

40. Alessi A, Brandao AA, Coca A, Cordeiro AC, Nogueira AR, Diogenes de Magalhaes $\mathrm{F}$, et al. First Brazilian position on resistant hypertension. Arq Bras Cardiol. 2012;99(1):576-85.

41. Souza WKS, Jardim PCB, Brito LP, Araújo FA, Sousa ALL. Automedida da pressão arterial para o controle das cifras tensionais e para a adesão ao tratamento. Arq Bras Cardiol. 2012;98:(2)167-74.

42. Vamsi V, Golub A, Mija P, Fekete P, Findri F, Prkacin I. Central blood pressure and pulse wave velocity in patients with resistant hypertension. Signa Vitae. 2018;14(Suppl 1):28-30.

43. Cuspidi C, Macca G, Sampieri L, Michev I, Salerno M, Fusi V, et al. High prevalence of cardiac and extracardiac target organ damage in refractory hypertension. J Hypertens. 2001;19(11):2063-70.

44. de la Sierra A, Banegas JR, Oliveras A, Gorostidi M, Segura J, de la Cruz IJ, et al. Clinical differences between resistant hypertensives and patients treated and controlled with three or less drugs. J Hypertens. 2012;30(6):1211-6.

45. Sim JJ, Bhandari SK, Shi J, Reynolds K, Calhoun DA, Kalantar-Zadeh K, et al. Comparative risk of renal, cardiovascular, and mortality outcomes in controlled, uncontrolled resistant, and nonresistant hypertension. Kidney Int. 2015;88(3):622-32.

46. Nilsson PM. Hemodynamic Aging as the Consequence of Structural Changes Associated with Early Vascular Aging (EVA). Aging Dis. 2014;5(2):109-13.

47. Papakatsika S, Stabouli S, Antza C, Kotsis V. Early Vascular Aging: A New Target for Hypertension Treatment. Curr Pharm Des. 2016;22(1):122-6.

48. Barbaro NR, de Araujo TM, Tanus-Santos JE, Anhe GF, Fontana V, Moreno H. Vascular Damage in Resistant Hypertension: TNF-Alpha Inhibition Effects on Endothelial Cells. Biomed Res Int. 2015;2015:631594.

49. Barbaro NR, Fontana V, Modolo R, De Faria AP, Sabbatini AR, Fonseca FH, et al. Increased arterial stiffness in resistant hypertension is associated with inflammatory biomarkers. Blood Pressure.2015;24(1):7-13.

50. Camici GG, Savarese G, Akhmedov A, Luscher TF. Molecular mechanism of endothelial and vascular aging: implications for cardiovascular disease. Eur HeartJ. 2015;36(48):3392-403.

51. Harvey A, Montezano AC, Touyz RM. Vascular biology of ageing-Implications in hypertension. J Mol Cell Cardiol. 2015 Jun;83:112-21.

52. Rizzoni D, Agabiti Rosei C, DeCiuceis C, SemeraroF, Rizzoni M, Docchio F.New Methods to Study the Microcirculation. Am J Hypertens. 2018;31(3):265-73.

53. Lehmann MV, Schmieder RE. Remodeling of retinal small arteries in hypertension. Am J Hypertens. 2011;24(12):1267-73.

54. Cecelja M, ChowienczykP.Molecular Mechanisms of Arterial Stiffening. Pulse (Basel). 2016;4(1):43-8.

55. Mikael LR, Paiva AMG, Gomes MM, Sousa ALL, Jardim P, Vitorino PVO, et al. Vascular Aging and Arterial Stiffness. Arq Bras Cardiol. 2017;109(3):253-8.

56. Mozos I, Malainer C, Horbanczuk J, Gug C, Stoian D, Luca CT, et al. Inflammatory Markers for Arterial Stiffness in Cardiovascular Diseases. Front Immunol. 2017;8:1058

57. Balduino Mendes AB, Giollo-Junior LT, de Andrade DO, Gregorio ML, YugarToledo JC, Vilela-MartinJF. How to Investigate the VascularChanges in Resistant Hypertension. Curr Hypertens Rev. 2016;12(2):139-47.

58. Virdis A. Arterial Stiffness and Vascular Aging: From Pathophysiology to Treatment, with a Look at the Future. High Blood Press Cardiovasc Prev. 2018;25(2):135-6.

59. Joly L. Arterial stiffness and cognitive function. Geriatr Psychol Neuropsychiatr Vieil. 2017;15(1):83-8.

60. Hughes TM, Wagenknecht LE, Craft S, Mintz A, Heiss G, Palta P, et al. Arterial stiffness and dementia pathology: Atherosclerosis Risk in Communities (ARIC)PET Study. Neurology. 2018;90(14):e1248-e56.
61. Muntner P, Davis BR, Cushman WC, Bangalore S, Calhoun DA, Pressel SL, et al. Treatment-resistant hypertension and the incidence of cardiovascular disease and end-stage renal disease: results from the Antihypertensive and Lipid-Lowering Treatmentto Prevent HeartAttack Trial (ALLHAT). Hypertension. 2014;64(5):1012-21.

62. Lotufo PA, Pereira AC, Vasconcellos PS, Santos IS, Mill JG, Bensenor IM. Resistant Hypertension: Risk Factors, Subclinical Atherosclerosis, and Comorbidities Among Adults—-The Brazilian Longitudinal Study of Adult Health (ELSA-Brasil). J Clin Hypertens. 2015;17(1):74-80.

63. ladecola C. Hypertension and Dementia. Hypertension. 2014;64(1):3-5.

64. LaurentS, BoutouyrieP.Thestructural factor of hypertension: largeand small artery alterations. Circ Res. 2015;116(6):1007-21.

65. Howard VJ, Tanner RM, Anderson A, Invin MR, Calhoun DA, Lackland DT, etal. Apparent Treatment-resistant Hypertension Among Individuals with History of Stroke or Transient Ischemic Attack. Am J Med. 2015;128(7):707-14.e2.

66. Hong J-H, Sohn S-I, Kwak J, Yoo J, Ahn SJ, Woo SJ, et al. Retinal artery occlusion and associated recurrent vascular risk with underlying etiologies. PLoS One. 2017;12(6):e0177663.

67. HongD, StradlingD, DasturCK, AkbariY, Groysman L,Al-Khoury L, etal. Resistant Hypertension after Hypertensive Intracerebral Hemorrhage Is Associated with More Medical Interventions and Longer Hospital Stays without Affecting Outcome. Front Neurol. 2017;8:184

68. Chen Y, Shen F, Liu J, Yang GY. Arterial stiffness and stroke: de-stiffening strategy, a therapeutic target for stroke. Stroke Vasc Neurol. 2017; 2(2):65-72.

69. CuspidiC, VaccarellaA, Negri F, Sala C. Resistanthypertension and leftventricular hypertrophy: an overview. J Am Soc Hypertens. 2010;4(6):319-24.

70. Nadruz W. Myocardial remodeling in hypertension. J Hum Hypertens. 2015;29(1):1-6

71. Muxfeldt ES, Salles GF. Pulse pressure or dipping pattern: which one is a better cardiovascular risk marker in resistant hypertension? J Hypertens. 2008;26(5):878-84.

72. Modolo R, de FariaAP,PaganelliMO, SabbatiniAR, Barbaro NR, Nascimento BB, et al. Predictors of silent myocardial ischemia in resistant hypertensive patients. AmJ Hypertens. 2015;28(2):200-7.

73. Muxfeldt ES, de Souza F, Margallo VS, Salles GF. Cardiovascular and renal complications in patients with resistant hypertension. Curr Hypertens Rep. 2014;16(9):471.

74. NadruzW, Shah AM, Solomon SD. Diastolic Dysfunction and Hypertension. Med Clin North Am. 2017;101(1):7-17.

75. Rodrigues CIS CR, Almeida FA. Hipertensao arterial resistente. In: Moura LRR, Alves MAR, Santos DR, Pecoits Filho R. Tratado de Nefrologia. São Paulo: Atheneu; 2018.

76. Summary of Recommendation Statements. Kidney Int Suppl.2013;3(1):5-14.

77. TalerSJ, TextorSC,AugustineJE. Resistanthypertension: comparinghemodynamic management to specialist care. Hypertension. 2002;39(5):982-8.

78. Gaddam KK, Nishizaka MK, Pratt-Ubunama MN, Pimenta E, Aban I, Oparil S, et al. Characterization of resistant hypertension: association between resistant hypertension, aldosterone, and persistent intravascular volume expansion. Arch Intern Med. 2008;168(11):1159-64.

79. Pimenta E, Gaddam KK, Oparil S, Aban I, Husain S, Dell'Italia LJ, et al. Effects of dietary sodium reduction on blood pressure in subjects with resistant hypertension: resultsfrom a randomized trial. Hypertension. 2009;54(3):475-81.

80. Agarwal R. Resistant hypertension and the neglected antihypertensive: sodium restriction. Nephrol Dial Transplant. 2012;27(11):4041-5.

81. Shimosawa T. Salt, the renin-angiotensin-aldosterone system and resistant hypertension. Hypertens Res. 2013;36(8):657-60.

82. Calhoun DA. Refractoryand Resistant Hypertension: Anthypertensive Treatment Failure versus Treatment Resistance. Korean Circ J. 2016;46(5):593-600.

83. Eirin A, Textor SC, Lerman LO. Emerging concepts for patients with treatmentresistant hypertension. Trends Cardiovasc Med. 2016;26(8):700-6. 
84. Eide IK, Torjesen PA, Drolsum A, Babovic A, Lilledahl NP. Low-renin status in therapy-resistant hypertension: a clue to efficient treatment. J Hypertens. 2004;22(11):2217-26.

85. Laragh JH, Sealey JE. The Plasma Renin Test Reveals the Contribution of Body Sodium-Volume Content (V) and Renin-Angiotensin (R) Vasoconstriction to Long-Term Blood Pressure. Am J Hypertens. 2011;24(11):1164-80

86. Calhoun DA, Booth JN, 3rd, Oparil S, Irvin MR, Shimbo D, Lackland DT, et al. Refractory hypertension: determination of prevalence, risk factors, and comorbidities in a large, population-based cohort. Hypertension. 2014;63(3):451-8

87. Judd EK, Calhoun DA, Warnock DG. Pathophysiology and Treatment of Resistant Hypertension: The Role of Aldosterone and Amiloride-Sensitive Sodium Channels. Semin Nephrol. 2014;34(5):532-9.

88. Tsioufis C, Kordalis A, Flessas D, Anastasopoulos I, Tsiachris D, Papademetriou V, et al. Pathophysiology of resistant hypertension: the role of sympathetic nervous system. Int J Hypertens. 2011;2011:642416.

89. Dudenbostel T, Acelajado MC, Pisoni R, Li P, Oparil S, Calhoun DA. Refractory Hypertension: Evidence of Heightened Sympathetic Activity as a Cause of Antihypertensive Treatment Failure. Hypertension. 2015;66(1):126-33

90. Mahmud A, Mahgoub M, Hall M, Feely J. Does aldosterone-to-renin ratio predict the antihypertensive effect of the aldosterone antagonist spironolactone? Am J Hypertens. 2005;18(12 Pt 1):1631-5.

91. Pimenta E, Calhoun DA. Resistant hypertension and aldosteronism. Curr Hypertens Rep. 2007;9(5):353-9.

92. Wang C, Xiong B, HuangJ. Efficacy and Safety of Spironolactone in Patients with Resistant Hypertension: A Meta-analysis of Randomised Controlled Trials. Heart Lung Circ. 2016;25(10):1021-30.

93. Jain S, Khera R, Corrales-Medina VF, Townsend RR, Chirinos JA. "Inflammation and arterial stiffness in humans". Atherosclerosis. 2014;237(2):381-90.

94. Pedrosa RP, Drager LF, Gonzaga CC, Sousa MG, de Paula LK, Amaro AC, et al. Obstructive sleep apnea: the most common secondary cause of hypertension associated with resistant hypertension. Hypertension. 2011;58(5):811-7.

95. Florczak E, Prejbisz A, Szwench-Pietrasz E, Sliwinski P, Bielen P, Klisiewicz $A$, et al. Clinical characteristics of patients with resistant hypertension: the RESIST-POL study. J Hum Hypertens. 2013;27(11):678-85.

96. de Faria AP, Modolo R, Fontana V, Moreno H. Adipokines: novel players in resistant hypertension. J Clin Hypertens (Greenwich). 2014;16(10):754-9.

97. Lacerda L, Faria AP, Fontana V, Moreno H, Sandrim V. Role of MMP-2 and MMP-9 in resistance to drug therapy in patients with resistant hypertension. Arq Bras Cardiol. 2015;105(2):168-75.

98. de Faria AP, Ritter AM, Sabbatini AR, Correa NB, Brunelli V, Modolo R, et al. Deregulation of Soluble Adhesion Molecules in Resistant Hypertension and Its Role in Cardiovascular Remodeling. Circ J. 2016;80(5):1196-201 .

99. Sabbatini AR, Barbaro NR, de Faria AP, Ritter AMV, Modolo R, Correa NB, et al. Matrix metalloproteinase- $2-735 \mathrm{C} / \mathrm{T}$ polymorphism is associated with resistant hypertension in a specialized outpatient clinic in Brazil. Gene. 2017;620:23-9

100. Lacchini R, Sabha M, Coeli FB, Favero FF, Yugar-Toledo J, Izidoro-Toledo TC, et al. T allele of $-344 \mathrm{C} / \mathrm{T}$ polymorphism in aldosterone synthase gene is not associated with resistant hypertension. Hypertens Res. 2009;32(2):159-62

101. Yugar-Toledo JC, Martin JF, Krieger JE, Pereira AC, Demacq C, Coelho OR, et al. Gene variation in resistant hypertension: multilocus analysis of the angiotensin 1-converting enzyme, angiotensinogen, and endothelial nitric oxide synthase genes. DNA Cell Biol. 2011;30(8):555-64 .
102. Figueiredo VN, Yugar-Toledo JC, Martins LC, Martins LB, de Faria AP, de Haro Moraes $\mathrm{C}$, et al. Vascular stiffness and endothelial dysfunction Correlations at different levels of blood pressure. Blood Press. 2012;21(1):31-8

103. Quinaglia T, Martins LC, Figueiredo VN, Santos RC, Yugar-Toledo JC, Martin JF, et al. Non-dipping pattern relates to endothelial dysfunction in patients with uncontrolled resistant hypertension. J Hum Hypertens. 2011;25(11):656-64

104. Pierdomenico SD, Lapenna D, Bucci A, Di Tommaso R, Di Mascio R, Manente BM, et al. Cardiovascular outcome in treated hypertensive patients with responder, masked, false resistant, and true resistant hypertension. Am J Hypertens. 2005;18(11):1422-8.

105. Rimoldi SF, Scherrer U, Messerli FH. Secondary arterial hypertension: when, who, and how to screen? Eur Heart J. 2014;35(19):1245-54.

106. Braam B, Taler SJ, Rahman M, Fillaus JA, Greco BA, Forman JP, et al. Recognition and Management of Resistant Hypertension. Clin J Am Soc Nephrol. 2017;12(3):524-35.

107. Hou H, Zhao Y, Yu W, Dong H, Xue X, Ding J, et al. Association of obstructive sleep apnea with hypertension: A systematic review and metaanalysis. J Glob Health. 2018;8(1):010405.

108. Drager LF, Bortolotto LA, Lorenzi MC, Figueiredo AC, Krieger EM, LorenziFilho G. Early signs of atherosclerosis in obstructive sleep apnea. Am J Respir Crit Care Med. 2005;172(5):613-8.

109. Senaratna CV, Perret JL, Matheson MC, Lodge CJ, Lowe AJ, Cassim R, et al. Validity of the Berlin questionnaire in detecting obstructive sleep apnea: A systematic review and meta-analysis. Sleep Med Rev. 2017;36:116-24.

110. Fava C, Dorigoni S, Dalle Vedove F, Danese E, Montagnana M, Guidi GC, et al. Effect of CPAP on blood pressure in patients with OSA/hypopnea a systematic review and meta-analysis. Chest. 2014;145(4):762-71.

111. Guo J, Sun Y, Xue LJ, Huang ZY, Wang YS, Zhang L, et al. Effect of CPAP therapy on cardiovascular events and mortality in patients with obstructive sleep apnea: a meta-analysis. Sleep Breath. 2016;20(3):965-74.

112. Horl MP, Horl WH. Hemodialysis-associated hypertension: pathophysiology and therapy. Am J Kidney Dis. 2002;39(2):227-44.

113. Wolley MJ, Stowasser M. Resistant Hypertension and Chronic Kidney Disease: a Dangerous Liaison. Curr Hypertens Rep. 2016;18(5):36.

114. van Jaarsveld BC, Krijnen P, Pieterman H, Derkx FH, Deinum I, Postma CT, et al. The effect of balloon angioplasty on hypertension in atherosclerotic renal-artery stenosis. Dutch Renal Artery Stenosis Intervention Cooperative Study Group. N Engl J Med. 2000;342(14):1007-14.

115. Bavishi C, de Leeuw PW, Messerli FH. Atherosclerotic Renal Artery Stenosis and Hypertension: Pragmatism, Pitfalls, and Perspectives. Am J Med. 2016;129(6):635 e5- e14

116. Van der Niepen P, Rossignol P, Lengele JP, Berra E, Sarafidis P, Persu A. Renal Artery Stenosis in Patients with Resistant Hypertension: Stent It or Not? Curr Hypertens Rep. 2017; 19(1):5.

117. Mulherin JL, Jr., Edwards WH. Alternative methods of renal revascularization. Ann Surg. 1987;205(6):740-6.

118. Calhoun DA. Is there an unrecognized epidemic of primary aldosteronism? Pro. Hypertension. 2007;50(3):447-53; discussion -53.

119. Kline GA, Prebtani APH, Leung AA, Schiffrin EL. Primary aldosteronism: a common cause of resistant hypertension. CMAJ. 2017;189(22):E773-E8

120. Young JWF, Calhoun DA, Lenders JWM, Stowasser M, Textor SC. Screening for Endocrine Hypertension: An Endocrine Society Scientific Statement. Endocrine Rev. 2017;38(2):103-22.

121. Funder JW, Carey RM, Mantero F, Murad MH, Reincke M, Shibata H, etal. The Management of Primary Aldosteronism: Case Detection, Diagnosis, and Treatment: An Endocrine Society Clinical Practice Guideline. J Clin Endocrinol Metab. 2016;101(5):1889-916. 
122. Lenders JW, Eisenhofer G, Mannelli M, Pacak K. Phaeochromocytoma Lancet. 2005;366(9486):665-75.

123. Pillai S, Gopalan V, Smith RA, Lam AK. Updates on the genetics and the clinical impacts on phaeochromocytoma and paraganglioma in the new era. Crit Rev Oncol Hematol. 2016;100:190-208.

124. Naranjo J, Dodd S, Martin YN. Perioperative Management of Pheochromocytoma. J Cardiothorac Vasc Anesth. 2017;31(4):1427-39.

125. Saito I, Ito K, Saruta T. Hypothyroidism as a cause of hypertension. Hypertension. 1983;5(1):112-5.

126. Levey GS, Klein I. Catecholamine-thyroid hormone interactions and the cardiovascular manifestations of hyperthyroidism. Am J Med. 1990;88(6):642-6.

127. Lenders JW, Duh QY, Eisenhofer G, Gimenez-Roqueplo AP, Grebe SK, Murad MH, et al. Pheochromocytoma and paraganglioma: an endocrine society clinical practice guideline. J Clin Endocrinol Metab. 2014;99(6):1915-42.

128. Pappachan JM, Tun NN, Arunagirinathan G, Sodi R, Hanna FWF. Pheochromocytomas and Hypertension. Curr Hypertens Rep. 2018;20(1):3.

129. Kenny D, Polson JW, Martin RP, Paton JF, Wolf AR. Hypertension and coarctation of the aorta: an inevitable consequence of developmental pathophysiology. Hypertens Res. 2011;34(5):543-7.

130. Cohen JB. Hypertension in Obesity and the Impact of Weight Loss. Curr Cardiol Rep. 2017;19(10):98.

131. Lloyd-Jones DM, Evans JC, Larson MG, O'Donnell CJ, Roccella EJ, Levy D. Differential control of systolic and diastolic blood pressure : factors associated with lack of blood pressure control in the community. Hypertension. 2000;36(4):594-9.

132. Bramlage P, Pittrow D, Wittchen HU, Kirch W, Boehler S, Lehnert H, et al. Hypertension in overweight and obese primary care patients is highly prevalent and poorly controlled. Am J Hypertens. 2004;17(10):904-10.

133. Aucott L, Poobalan A, Smith WC, Avenell A, Jung R, Broom J. Effects of weight loss in overweight/obese individuals and long-term hypertension outcomes: a systematic review. Hypertension. 2005;45(6):1035-41.

134. Schiavon CA, Bersch-Ferreira AC, Santucci EV, Oliveira JD, Torreglosa CR, Bueno PT, et al. Effects of Bariatric Surgery in Obese Patients With Hypertension: The GATEWAY Randomized Trial (Gastric Bypass to Treat Obese Patients With Steady Hypertension). Circulation. 2018;137(11):1132-42

135. Edwards DG, Farquhar WB. Vascular effects of dietary salt. Curr Opin Nephrol Hypertens. 2015;24(1):8-13.

136. White WB, Turner JR, Sica DA, Bisognano JD, Calhoun DA, Townsend $R R$, et al. Detection, evaluation, and treatment of severe and resistant hypertension. In: Congress of American Society of Hypertension Interactive (forum) Bethesda, MD, October 10th 2013. Proceedings. J Am Soc Hypertens. 2014;8(10):743-57.

137. He FJ, Li J, Macgregor GA. Effect of longer term modest salt reduction on blood pressure: Cochrane systematic review and meta-analysis of randomised trials. BMJ. 2013;346:f1325.

138. Wildman RP, Gu D, Muntner P, Huang G, Chen J, Duan X, et al. Alcohol intake and hypertension subtypes in Chinese men. J Hypertens. 2005;23(4):737-43.

139. Sarwar MS, Islam MS, Al Baker SM, Hasnat A. Resistant hypertension: underlying causes and treatment. Drug Res (Stuttg). 2013;63(5):217-23.

140. Roerecke M, Kaczorowski J, Tobe SW, Gmel G, Hasan OSM Rehm J. The effect of a reduction in alcohol consumption on blood pressure: a systematic review and meta-analysis. Lancet Publ Health. 2017;2(2):e108-e20.

141. Ribeiro F, Costa R, Mesquita-Bastos J. Exercise training in the management of patients with resistant hypertension. World J Cardiol. 2015;7(2):47-51.
142. Dimeo F, Pagonas N, Seibert F, Arndt R, Zidek W, Westhoff TH. Aerobic exercise reduces blood pressure in resistant hypertension. Hypertension. 2012;60(3):653-8.

143. Guimaraes GV, de Barros Cruz LG, Fernandes-Silva MM, Dorea EL, Bocchi EA. Heated water-based exercise training reduces 24-hour ambulatory blood pressure levels in resistant hypertensive patients: a randomized controlled trial (HEx trial). Int J Cardiol. 2014;172(2):434-41.

144. Santos LP, Moraes RS, Vieira PJ, Ash GI, Waclawovsky G, Pescatello LS, et al. Effects of aerobic exercise intensity on ambulatory blood pressure and vascular responses in resistant hypertension: a crossover trial. J Hypertens. 2016;34(7):1317-24.

145. Kruk PJ, Nowicki M. Effect of the physical activity program on the treatment of resistant hypertension in primary care. Prim Health Care Res Dev. 2018; 22:1-9.

146. Cruz LG, Bocchi EA, Grassi G, Guimaraes GV. Neurohumoral and Endothelial Responses to Heated Water-Based Exercise in Resistant Hypertensive Patients. Circ J. 2017;81(3):339-45.

147. Cornelissen VA, Fagard RH, Coeckelberghs E, Vanhees L. Impact of resistance training on blood pressure and other cardiovascular risk factors: a meta-analysis of randomized, controlled trials. Hypertension. 2011;58(5):950-8.

148. Narayan P, Doumas M, Kumar A, Faselis CJ, Myers JN, Pittaras A, et al. Impact of Cardiorespiratory Fitness on Mortality in Black Male Veterans With Resistant Systemic Hypertension. Am J Cardiol. 2017;120(9):1568-71.

149. Povoa R, Barroso WS, Brandao AA, Jardim PC, Barroso O, Passarelli O, Jr., et al. I brazilian position paper on antihypertensive drug combination. Arq Bras Cardiol. 2014;102(3):203-10.

150. Whelton PK, Carey RM, Aronow WS, Casey DE, Jr., Collins KJ, Dennison Himmelfarb C, et al. 2017 ACC/AHA/AAPA/ABC/ACPM/AGS/APhA/ASH/ ASPC/NMA/PCNA Guideline for the Prevention, Detection, Evaluation, and Management of High Blood Pressure in Adults: A Report of the American College of Cardiology/American Heart Association Task Force on Clinical Practice Guidelines. Hypertension. 2018;71(6):e13-e115.

151. Salles GF, Cardoso CR, Fonseca LL, Fiszman R, Muxfeldt ES. Prognostic significance of baseline heart rate and its interaction with betablocker use in resistant hypertension: a cohort study. Am J Hypertens. $2013 ; 26(2): 218-26$

152. Williams B, MacDonald TM, Morant S, Webb DJ, Sever P, McInnes $\mathrm{G}$, et al. Spironolactone versus placebo, bisoprolol, and doxazosin to determine the optimal treatment for drug-resistant hypertension (PATHWAY-2): a randomised, double-blind, crossover trial. Lancet. 2015;386(10008):2059-68.

153. Liu G, Zheng XX, Xu YL, Lu J, Hui RT, Huang XH. Effect of aldosterone antagonists on blood pressure in patients with resistant hypertension: a meta-analysis. J Hum Hypertens. 2015;29(3):159-66.

154. Dahal K, Kunwar S, Rijal J, Alqatahni F, Panta R, Ishak N, et al. The Effects of Aldosterone Antagonists in Patients With Resistant Hypertension: A MetaAnalysis of Randomized and Nonrandomized Studies. Am J Hypertens. 2015;28(11):1376-85.

155. Williams B, MacDonald TM, Morant SV, Webb DJ, Sever P, McInnes GT, et al. Endocrine and haemodynamic changes in resistant hypertension, and blood pressure responses to spironolactone or amiloride: the PATHWAY-2 mechanisms substudies. Lancet Diabetes Endocrinol. 2018;6(6):464-75.

156. van Kleef MEAM, Bates MC, Spiering W. Endovascular Baroreflex Amplification for Resistant Hypertension. Curr Hypertens Report. 2018;20(5):46.

157. Bisognano JD, Bakris G, Nadim MK, Sanchez L, Kroon AA, Schafer $J$, et al. Baroreflex activation therapy lowers blood pressure in patients with resistant hypertension: results from the double-blind, randomized, placebo-controlled rheos pivotal trial. J Am Coll Cardiol. 2011;58(7):765-73. 
158. Heusser K, Tank J, Brinkmann J, Menne J, Kaufeld J, Linnenweber-Held $\mathrm{S}$, et al. Acute Response to Unilateral Unipolar Electrical Carotid Sinus Stimulation in Patients with Resistant Arterial Hypertension. Hypertension. 2016;67(3):585-91

159. Wallbach M, Lehnig LY, Schroer C, Luders S, Bohning E, Muller GA, et al. Effects of Baroreflex Activation Therapy on Ambulatory Blood Pressure in Patients With Resistant Hypertension. Hypertension. 2016;67(4):701-9.

160. Schmieder RE, Mahfoud F, Azizi M, Pathak A, Dimitriadis K, Kroon AA, et al. European Society of Hypertension position paper on renal denervation. J Hypertens. 2018;391(10137):2335-45.

161. Oparil S, Schmieder RE. New approaches in the treatment of hypertension. Circ Res. 2015;116(6):1074-95.

162. Bakris GL, Townsend RR, Flack JM, Brar S, Cohen SA, D'Agostino R, et al. 12-month blood pressure results of catheter-based renal artery denervation for resistant hypertension: the SYMPLICITY HTN-3 trial. J Am Coll Cardiol. 2015;65(13):1314-21.

163. Pappaccogli M, Covella M, Berra E, Fulcheri C, Di Monaco S, Perlo E, et al. Effectiveness of Renal Denervation in Resistant Hypertension: A Meta-Analysis of 11 Controlled Studies. High Blood Press Cardiovasc Prev. 2018;25(2):167-76.

164. Morganti A, Mancia G. Resistant hypertension: Renal denervation or intensified medical treatment? Eur J Intern Med. 2018;50:6-11.

165. Lozano L, Tovar JL, Sampol G, Romero O, Jurado MJ, Segarra A, et al Continuous positive airway pressure treatment in sleep apnea patients with resistant hypertension: a randomized, controlled trial. J Hypertens. $2010 ; 28(10): 2161-8$.

166. Pedrosa RP, Drager LF, de Paula LKG, Amaro ACS, Bortolotto LA, Lorenzi-Filho G. Effects of OSA treatment on BP in patients with resistant hypertension: a randomized trial. Chest. 2013;144(5):1487-94.

167. Martinez-Garcia MA, Capote F, Campos-Rodriguez F, Lloberes P, Diaz de Atauri MJ, Somoza M, et al. Effect of CPAP on blood pressure in patients with obstructive sleep apnea and resistant hypertension: the HIPARCO randomized clinical trial. JAMA. 2013;310(22):2407-15.

168. de Oliveira AC, Martinez D, Massierer D, Gus M, Goncalves SC, Ghizzoni $\mathrm{F}$, et al. The antihypertensive effect of positive airway pressure on resistant hypertension of patients with obstructive sleep apnea: a randomized, double-blind, clinical trial. Am J Respir Crit Care Med. 2014;190(3):345-7.

169. Muxfeldt ES, Margallo V, Costa LM, Guimaraes G, Cavalcante AH, Azevedo JC, et al. Effects of continuous positive airway pressure treatment on clinic and ambulatory blood pressures in patients with obstructive sleep apnea and resistant hypertension: a randomized controlled trial. Hypertension. 2015;65(4):736-42.

170. Litvin AY, Sukmarova ZN, Elfimova EM, Aksenova AV, Galitsin PV Rogoza AN, et al. Effects of CPAP on "vascular" risk factors in patients with obstructive sleep apnea and arterial hypertension. Vasc Health Risk Manag. 2013;9:229-35.

171. Joyeux-Faure M, Baguet JP, Barone-Rochette G, Faure P, Sosner P, MounierVehier C, et al. Continuous Positive Airway Pressure Reduces Night-Time Blood Pressure and Heart Rate in Patients With Obstructive Sleep Apnea and Resistant Hypertension: The RHOOSAS Randomized Controlled Trial. Front Neurol. 2018;9:318.

172. Sanchez-de-la-Torre M, Khalyfa A, Sanchez-de-la-Torre A, MartinezAlonso M, Martinez-Garcia MA, Barcelo A, et al. Precision Medicine in Patients With Resistant Hypertension and Obstructive Sleep Apnea: Blood Pressure Response to Continuous Positive Airway Pressure Treatment. J Am Coll Cardiol. 2015;66(9):1023-32.

173. Bertog SC, Sobotka NA, Sobotka PA, Lobo MD, Sievert K, Vaskelyte L, et al. Percutaneous Creation of a Central Iliac Arteriovenous Anastomosis for the Treatment of Arterial Hypertension. Curr Hypertens Rep. 2018;20(3): 18

174. Lobo MD, Sobotka PA, Stanton A, Cockcroft JR, Sulke N, Dolan E, et al. Central arteriovenous anastomosis for the treatment of patients with uncontrolled hypertension (the ROX CONTROL HTN study): a randomised controlled trial. Lancet. 2015;385(9978):1634-41. 